\title{
New families of optimal high-energy ternary sequences having good correlation properties
}

\author{
Jeffrey R. Hollon ${ }^{1} \cdot$ Muralidhar Rangaswamy ${ }^{2} \cdot$ Pawan Setlur $^{3}$ \\ Received: 7 May 2018 / Accepted: 6 August 2018 / Published online: 20 August 2018 \\ (c) Springer Science+Business Media, LLC, part of Springer Nature 2018
}

\begin{abstract}
We employ algebraic methods to provide some new constructions of what we call optimal high-energy ternary sequences (a sequence with entries in $\{0,1,-1\}$ with a single zero, having optimal correlation properties). Our motivation for these constructions stems from their usefulness in several areas related to communication and radar systems.
\end{abstract}

Keywords Sequences · Difference sets · Correlation · Partial difference sets

\section{Introduction}

Sequences with ideal autocorrelation properties have many applications in spread spectrum communication systems such as code division multiple access (CDMA) systems, radar, signal processing, and source coding (audio, video or both etc.). Such sequences find many applications in signal processing and serve as aperture weighting functions in electromagnetic and acoustic imaging [7].

Sequences and their higher-dimensional counterparts (arrays) are critical in today's technological world where they are used in radar, error correction, digital communication, etc. Our work so far has resulted in the discovery of a few very rich classes of sequences all of whose out-of-phase autocorrelation values (side lobes) are very small. We call the constructed sequences as perfect sequences, and they serve as perfect algebraic/combinatorial objects in designing signals for communication and radar applications. Our work would be applicable in a much larger arena, opening doors for new application areas in the MIMO radar study as well.

$凶 \quad$ Jeffrey R. Hollon

jeffhollon@gmail.com

1 Department of Mathematics and Statistics, Wright State University, Dayton, OH 45435, USA

2 Air Force Research Lab, Wright Patterson Air Force Base, Building 620, 2241 Avionics Circle, Dayton, OH 45433-7132, USA

3 Radar Assessment and Development Lab, Riverside Research, Beavercreek, OH 45431, USA 
In this paper, we employ algebraic methods to investigate perfect sequences and perfect arrays (binary and ternary) and construct what we call high-energy ternary sequences (a sequence with entries in $\{0,1,-1\}$ with a single zero). Our motivation stems from their usefulness in several areas related to communication and radar systems: quantum computing, MC-CDMA systems, quasi-synchronous CDMA, multiple antenna wireless communication systems, FHSS which are widely used in military radios, CDMA and GSM networks, radars and sonars, and Bluetooth communications to name a few.

Our new sequences possess very desirable correlation properties:

1. The peak side lobes in the autocorrelation are small.

2. The sum of the squares of the side lobes in the autocorrelation is small.

Their ambiguity functions and Doppler tolerance [12] suggest that they are suitable for radar applications. While binary sequences/signals are typically sought after (having all its entries as 1 or -1 ), we only make a very modest assumption: allowing a SINGLE zero, thereby making them almost binary and achieving close to $100 \%$ energy. The gain is astounding: The sum of the squares of the side lobes in the autocorrelation is cut down by $50 \%$. There are cases where we get other waveforms which are close to optimal but still look promising for radar use. Preliminary simulations validate our claim. Extension to two zeros is possible in a straightforward manner.

In Sect. 2, we provide basic definitions and algebraic preliminaries that pertain to group rings and combinatorial structures like Paley difference sets and their partial difference sets counterparts. Section 3 deals with our new constructions of what we call "optimal high-energy ternary sequences." The adjective "optimal" pertains to attaining the theoretical minimum sum of squares of the side lobes in the autocorrelation; "high energy" qualifier refers to "almost" binary since only a single zero is present in our new sequences. We lay the foundation by starting from the more general class of abelian difference sets and partial differences and then obtaining as special cases that arise from the aforementioned classical Paley-type objects. Circulant conference matrices are known not to exist. Interestingly enough, in Sect. 3, we exhibit an almost-cyclically developed conference matrix using our OHETS sequences.

The results we have obtained for sequences generalize nicely to the more general domain of arrays. It is our hope that the rapid advancement of technology will soon allow for arrays to be used alongside sequences in various engineering applications. The advantage of arrays, over sequences, is that they embrace a much larger spectrum of parameters (e.g., size and energy of the array). In Sect. 4, we define some performance measures for sequences and compare these measures with ours vs previously known ones.

Section 5 improves upon and provides a modification of a patented algorithm of McLaughlin to generate "good" sets of high-energy sequences. We define a "good" set of sequences to have the properties: 1. Each sequence has a high Golay merit factor (GMF). 2. The aperiodic autocorrelation side lobes of each sequence are small. These properties make them suitable for MIMO radar applications. An application of our OHETS to frame synchronization is given in Sect. 6. In Sect. 7, we conclude with some remarks and open problems. 


\section{Preliminaries}

A sequence $\mathbf{a}=\left(a_{i}\right)$, where $i=0,1,2 \ldots, N-1$, is called periodic with period $N$ provided that $a_{i}=a_{i+N}$ for all $i$. In this paper, we only consider real-valued sequences most of which will in fact be binary (respectively, ternary). That is all of their entries are either $\{+1,-1\}$ (respectively, $\{0,+1,-1\}$ ). The periodic cross-correlation function of the sequences $\mathbf{a}$ and $\mathbf{b}$ is defined by:

$$
C(\tau)=\sum_{i=0}^{N-1} a_{i} b_{(i+\tau) \bmod N}^{*}
$$

where $b_{i}^{*}$ represents the complex conjugate of $b_{i}$. In this definition, if $\mathbf{a}=\mathbf{b}$, we call it the periodic autocorrelation function (ACF) of $\mathbf{a}$. Note that the sequence $C=C(t)$ is again periodic with period $N$, so that it suffices to consider the autocorrelation coefficients $C(t)$ for $t \in\{0,1, \ldots, N-1\}$. The ACF measures how much the original sequence differs from its translates. The sequence $\mathbf{a}$ is said to have two-level ACF if its periodic $\mathrm{ACF}, C$, satisfies:

$$
C(\tau)= \begin{cases}E, & \tau=0 \\ F, & \tau \neq 0\end{cases}
$$

where $E$ and $F$ are two constants, respectively, denoting the in-phase and out-phase ACF of the sequence a. Let $\mathbf{a}=\left(a_{i}\right)$ be a binary sequence of period $N$. Define $D=\left\{0 \leq i \leq N-1: a_{i}=1\right\}$ and $d_{D}(t)=|(t+D) \cap D|$ which is called the difference function of $D \subseteq Z_{N}$. Then

$$
C(t)=N-4\left(k-d_{D}(t)\right)
$$

where $k=|D|$. This would serve as a bridge between binary sequences and combinatorial designs. From Eq. (3), we readily get the following:

Proposition $1 C(t) \equiv N(\bmod 4)$

We call $C(N), C(2 N), C(3 N), \ldots$ the "main lobes" and the remaining $C(i)$ as "side lobes." The sequence a is considered to have " $N$ elements," one main lobe and $N-1$ side lobes. It is well known that a sequence is said to have "good matched autocorrelation properties" if it satisfies:

1. The peak side lobes in the autocorrelation are small.

2. The sum of the squares of the side lobes in the autocorrelation is small.

Definition 1 Let $G$ be the additively written cyclic group with $v$ elements, (i.e., $G=$ $\left.Z_{v}\right)$ and $D$ a subset of $G$ with $k$ elements. For any $\alpha \neq 0(\bmod v)$, if the equation

$$
d-d^{\prime} \equiv \alpha(\bmod v)
$$

has exactly $\lambda$ solution pairs $\left(d, d^{\prime}\right)$ with both $d$ and $d^{\prime}$ in $D$, then the set $D$ is called a $(v, k, \lambda)$ difference set in $G$. 
The set $D$ defined in Definition 1 would work as the required cyclic difference set in the following result which is easy to prove.

Proposition 2 A periodic binary sequence with period $v$, $k$ entries +1 per period and 2-level ACF (with all nontrivial autocorrelation coefficients equal to $r$ ) is equivalent to a cyclic $(v, k, \lambda)$ difference set where $r=v-4(k-\lambda)$.

The case where $r=0$ corresponds to circulant Hadamard matrices of order $v$, where $v=4 u^{2}$, which are equivalent to cyclic $\left(4 u^{2}, 2 u^{2}-u, u^{2}-u\right)$ difference sets. The only known example of a circulant Hadamard matrix is when $v=4$ and it is conjectured that there are no others which exist. Likewise, the cases $r=1$ and $r=2$ give rise to only a handful of cyclic $(v, k, \lambda)$ difference sets. The case $r=-1$ would take us to a very fertile terrain where the examples are bountiful. In view of Proposition 2, these perfect binary sequences with $r=-1$ are equivalent to cyclic difference sets with parameters $\left(v, \frac{v-1}{2}, \frac{v-3}{4}\right)$. These are commonly referred to as Paley-Hadamard difference sets. The famous m-sequences, also referred to as singer sequences, provide a large such class.

The variation we wish to employ in this research is to allow a single zero in the sequences, making them ternary and obtaining new families of sequences with the desirable properties mentioned above for "good matched autocorrelation properties." The process of introducing a zero entry, where there was none before, we refer to as puncturing the sequence. A further property that these ternary sequences have is that of high energy, defined next.

Definition 2 The energy, $E$, of a sequence is calculated by $E=\sum_{i=0}^{N-1}\left|a_{i}\right|^{2}$.

Definition 3 The energy efficiency, $E_{\text {eff }}$, of a sequence is calculated by $E_{\text {eff }}=\frac{E}{N}$.

The sequences that are the main focus of this paper are ternary sequences with a single zero entry. Thus, the energy is maximized for ternary sequences (if the zero position were to be filled in, then we lose the ternary property). Further, it is desirable to have an energy efficiency which is close to 1 . This is also closely met by the same reasoning: For our sequences, with a single zero entry, $E_{\text {eff }}=\frac{N-1}{N}$ which approaches 1 as the sequence's period increases.

Perfect periodic autocorrelation sequences see applications in many areas, including spread spectrum communications [13], channel estimation and fast start-up equalization [10], pulse compression radars [5], sonar systems [16]. CDMA systems [6], system identification [15], and watermarking [14].

\subsection{Group ring notation}

We introduce the group ring notation which will be used in the proofs.

Definition 4 Let $G$ be a finite group and $R$ a ring where $G=\left\{g_{0}, g_{1}, g_{2}, \ldots, g_{n-1}\right\}$. Then, the group ring of $G$ over $R$ is the set denoted by $R[G]$ defined as:

$$
R[G]=\left\{\sum_{g \in G} a_{g} g \mid a_{g} \in R\right\} .
$$


$R[G]$ is also a free $R$-module of rank $n$ and an algebra. So, $R[G]$ is also referred to as a group algebra. When working with the group ring notation, multiplication and addition are defined in a way similar to polynomials, and the group elements are written multiplicatively. We further define the power of a group ring element in the following way.

Definition 5 If $W=\sum_{g \in G} a_{g} g$ is an element of $R[G]$ and $t$ some integer, then

$$
\left(\sum_{g \in G} a_{g} g\right)^{(t)}=\sum_{g \in G} a_{g} g^{t}
$$

The following is well known and follows readily from the above definition.

Lemma 1 Let $D=\left\{d_{0}, d_{1}, \ldots, d_{k-1}\right\}$. Then, $D$ is a $(v, k, \lambda)$ difference set if and only if

$$
D D^{(-1)}=k-\lambda+\lambda G \text { in } Z[G] .
$$

Remark Difference sets are studied in the more general group theoretic context. Since we are primarily dealing with "sequences," we restrict our attention to "cyclic" groups $Z_{v}$. We use the term "array" for when the group in question is non-cyclic. For more on these and related studies that pertain to sequences and arrays and their interplay with group developed combinatorial designs refer to the survey article [1].

\subsection{Abstraction of sequences to arrays}

In most of this paper, we are concerned with sequences. However, it is important to also consider more general, higher-dimensional objects, called arrays for which the theorems can be generalized to. An $r$-dimensional matrix, $A=\left(a\left(j_{1}, j_{2}, \ldots, j_{r}\right)\right)$, with $0 \leq j_{i} \leq s_{i}-1$ for $i=1,2, \ldots, r$, is called an $s_{1} \times s_{2} \times \cdots \times s_{r}$ array, where $s_{1}, s_{2}, \ldots, s_{r}$ are all positive integers. $A\left(j_{1}, j_{2}, \ldots, j_{r}\right)$ would denote the entry $a\left(j_{1}, j_{2}, \ldots, j_{r}\right)$ of the matrix $A$ at position $\left(j_{1}, j_{2}, \ldots, j_{r}\right)$. We refer to this array as a sequence when $r=1$.

These arrays have a similarly defined periodic autocorrelation function to sequences. The autocorrelation function $\gamma$ at $\tau=\left(t_{1}, t_{2}, \ldots, t_{r}\right)$ is given by

$$
\gamma(\tau)=\sum_{j_{1}=0}^{s_{1}-1} \cdots \sum_{j_{r}=0}^{s_{r}-1} a\left(j_{1}, j_{2}, \ldots, j_{r}\right) \bar{a}\left(j_{1}+t_{1}, j_{2}+t_{2}, \ldots, j_{r}+t_{r}\right)
$$

where the $i$ th subscript is taken modulo $s_{i}$ and $\bar{a}$ denotes the complex conjugate of $a$. The array corresponds to the group ring element $R[G]$ where $G=Z_{s_{1}} \times Z_{s_{2}} \times$ $\cdots \times Z_{s_{r}}=<g_{1}>\times<g_{2}>\times \cdots \times<g_{r}>$ and $g_{i}$ represents the generator of the cyclic group $Z_{s_{i}}$. 


\subsection{Difference sets of Paley type}

Example 1 Paley difference set for prime power $q \equiv 3(\bmod 4)$.

Let $q$ be a prime power and $q \equiv 3(\bmod 4)$. Let $G F(q)$ denote the finite field with $q$ elements. Fix a primitive element $\alpha$ of $G F(q)$ and define:

$$
\begin{aligned}
& S=\left\{\alpha^{2 i} \mid i=0,1, \ldots, \frac{q-3}{2}\right\}, \\
& N=\left\{\alpha^{2 j+1} \mid j=0,1, \ldots, \frac{q-3}{2}\right\} .
\end{aligned}
$$

The sets $S$ and $N$ consist exactly of the square and non-square elements of $G F(q) \backslash\{0\}$. It is well known that $S$ and $N$ are themselves difference sets in the group $G=$ $(G F(q),+)$ with parameters $(v, k, \lambda)=\left(q, \frac{q-1}{2}, \frac{q-3}{4}\right)$. Hence, the following equations hold in the group ring $Z[G]$ :

$$
\begin{aligned}
S S^{(-1)} & =N N^{(-1)}=\left(\frac{q+1}{4}\right)+\left(\frac{q-3}{4}\right) G \\
N & =S^{(-1)} \\
S & =N^{(-1)} \\
1+S+N & =G .
\end{aligned}
$$

The next example gives rise to the so-called partial difference set.

Definition 6 Let $G$ be a multiplicatively written group of order $v$. A subset $D \subseteq G$ of size $k$ is said to be a $(v, k, \lambda, \mu)$ partial difference set (PDS) in $G$ if:

1. $1 \notin D$;

2. $D=D^{(-1)}$;

3. $D D^{(-1)}=D^{2}=k+\lambda D+\mu(G-D-1)$ in $Z G$.

Example 2 Paley partial difference set for prime power $q \equiv 1(\bmod 4)$

Let $q$ be a prime power, $q \equiv 1(\bmod 4)$. Let $G F(q)$ denote the finite field with $q$ elements. Fix a primitive element $\alpha$ of $G F(q)$ and define:

$$
\begin{aligned}
& S=\left\{\alpha^{2 i} \mid i=0,1, \ldots, \frac{q-3}{2}\right\}, \\
& N=\left\{\alpha^{2 j+1} \mid j=0,1, \ldots, \frac{q-3}{2}\right\} .
\end{aligned}
$$

Thus, $S$ and $N$ consist exactly of the square and non-square elements of $G F(q) \backslash\{0\}$. It is well known that $S$ and $N$ are themselves partial difference sets in the group $G=(G F(q),+)$ with parameters $(v, k, \lambda, \mu)=\left(q, \frac{q-1}{2}, \frac{q-5}{4}, \frac{q-1}{4}\right)$. Hence, the following equations hold in the group ring $Z[G]$ : 


$$
\begin{aligned}
S S^{(-1)} & =S^{2}=\left(\frac{q-1}{2}\right)+\left(\frac{q-5}{4}\right) S+\left(\frac{q-1}{4}\right) N \\
N N^{(-1)} & =N^{2}=\left(\frac{q-1}{2}\right)+\left(\frac{q-5}{4}\right) N+\left(\frac{q-1}{4}\right) S \\
S & =S^{(-1)} \\
N & =N^{(-1)} \\
1+S+N & =G .
\end{aligned}
$$

More on the Paley sequences from their original source can be found in [11].

\section{Main results}

\subsection{Optimal high-energy ternary sequences and arrays from difference sets and almost difference sets}

The following proof examines the properties of difference and partial difference sets which are associated with an abelian group $G$ and what occurs when a position, namely the identity, is punctured. We continue by examining each case separately. Note that in the following theorems, the term "sequence" refers to a cyclic group while "array" refers to an abelian group that is not cyclic. Two theorems are required for the completion of these high-energy sequences. The first is by Camion and Mann [4] and the second by Arasu et al. [3].

Theorem 1 (Camion and Mann, [4]) Let $D$ be an abelian $(v, k, \lambda)$ difference set in $G$ which satisfies $D+D^{(-1)}+1=G$ in $Z[G]$. Then, $v$ is a prime power with $v \equiv 3(\bmod 4)$. If $G$ is cyclic then $v$ is prime and $D$ consists of the nonzero squares or the non-squares in $G F(v)$.

For partial difference sets, their parameters have been characterized in the following theorem using well-known equivalence of PDSs and strongly regular Cayley graphs.

Theorem 2 (Arasu et al. [3]) Let $\Gamma$ be a strongly regular Cayley graph based on an abelian group $G$ with parameters $(v, k, \lambda, \mu)$ satisfying $\beta=\lambda-\mu=-1$. Then, up to complementation, $\Gamma$ is either:

1. Of Paley type, that is it has the parameters of the type $\left(v, \frac{v-1}{2}, \frac{v-5}{4}, \frac{v-1}{4}\right)$ or;

2. it has parameters $(243,22,1,2)$.

\subsubsection{Case: difference sets}

Theorem 3 Let $D$ be a $(v, k, \lambda)$ difference set in an abelian group $G$. Then, the sequence (array) defined by

$$
S=D-(G-D)+1
$$


is a high-energy sequence (array) with up to three periodic side lobes. In the special case where $D \cap D^{(-1)}=\emptyset$, the sequence (array) $S$ will have only a two-valued periodic autocorrelation. Namely, the main lobe $v-1$ with side lobes $v-4(k-\lambda)$.

Proof Let $D$ be a $(v, k, \lambda)$ difference set in an abelian group $G$. Then

$$
D D^{(-1)}=k-\lambda+\lambda G
$$

and its complement, $G \backslash D$, is a $(v, v-k, v-k+2 \lambda)$ satisfying

$$
(G-D)(G-D)^{(-1)}=k-\lambda+(v-2 k+\lambda) G .
$$

Without loss of generality, we assume that $1 \notin D$. Consider the sequence (array) defined by $S=D-(G-D)+1$ and examine its autocorrelation $S S^{(-1)}$.

$$
\begin{aligned}
\sum_{x \in G} C(x) x=S S^{(-1)} & =[(2 D+1)-G]\left[\left(2 D^{(-1)}+1\right)-G\right] \\
& =4 D D^{(-1)}+2 D+2 D^{(-1)}+1+[v-(4 k+2)] G \\
& =2 D+2 D^{(-1)}+1+4[(k-\lambda)+\lambda G]+(v-4 k-2) G \\
& =2 D+2 D^{(-1)}+4(k-\lambda)+1+(v-4 k+4 \lambda-2) G .
\end{aligned}
$$

This calculation shows that the side lobe values are dependent on the intersection of elements in $D$ and $D^{(-1)}$. In particular, take the side lobe corresponding to the sequence element $x$ and its side lobe value $C(x)$. By the previous calculation

$$
C(x)= \begin{cases}v-4(k-\lambda)-2, & \text { if } x \notin D \cup D^{(-1)}, \\ v-4(k-\lambda), & \text { if } x \in D \text { or } x \in D^{(-1)}, \\ v-4(k-\lambda)+2, & \text { if } x \in D \cap D^{(-1)}\end{cases}
$$

In the special case where $D$ satisfies $D+D^{(-1)}+1=G, D$ is referred to as a skew symmetric difference set, then all side lobes collapse into a single value. In particular, all side lobe values will be $v-4(k-\lambda)+2$. Further, it is shown by use of Theorem 1 that this can only occur when the difference set has Paley parameters.

Example 3 As an example of Theorem 3, take the famous singer difference set (msequence) of length 15 . This sequence is given by

$$
D=[+++-++--+-+----]
$$

and its periodic correlation can be calculated to be

$$
C(D)=[15--------------] .
$$


Using Theorem 3,

$$
S=[0++-++--+-+----]
$$

whose periodic correlation is four-valued. This correlation is calculated to be

$$
C(S)=[14--+-(-3)+--+(-3)-+--] .
$$

\subsubsection{Case: partial difference sets}

Theorem 4 Let $D$ be $a(v, k, \lambda, \mu)$ partial difference set in an abelian group $G$. Then, the sequence (array) defined by

$$
S=D-(G-D)+1
$$

is a high-energy sequence (array) with up to two periodic side lobes. In the special case where $\mu=\lambda+1$, the sequence (array) $S$ will have only a two-valued periodic autocorrelation function. Namely, the mainlobe $v-1$ with side lobes given by $v+$ $4(\mu-k)-2$ and $v+4(\lambda-k)+2$. In the special case where $\mu-\lambda=1$, then $a$ single-valued periodic side lobe will exist and the partial difference will either have Paley parameters or have the parameters $(243,22,1,2)$.

Proof Let $D$ be a $(v, k, \lambda, \mu)$ partial difference set in an abelian group $G$. Then

$$
D D^{(-1)}=D^{2}=k+\lambda D+\mu(G-D-1) .
$$

Without loss of generality, we assume that $1 \notin D$ and examine the sequence (array) defined by

$$
S=D-(G-D)+1 \text {. }
$$

Sequence $S$ is a high-energy sequence whose periodic autocorrelation, $S S^{(-1)}$, can be calculated by:

$$
\begin{aligned}
\sum_{x \in G} C(x) x=S S^{(-1)} & =[(2 D+1)-G]\left[\left(2 D^{(-1)}+1\right)-G\right] \\
& =4 D D^{(-1)}+2 D+2 D^{(-1)}+1+[v-(4 k+2)] G \\
& =4 D^{2}+2 D+2 D+1+[v-(4 k+2)] G \\
& =4 D^{2}+4 D+1+[v-(4 k+2)] G \\
& =4[k-\mu+(\lambda-\mu) D+\mu G]+4 D+1+[v-4 k-2] G \\
& =4(k-\mu)+1+[4(\lambda-\mu)+4] D+[v+4 \mu-4 k-2] G .
\end{aligned}
$$

This calculation shows that the side lobe values will take on one of two values. One for entries in $D$ and the other for when entries are not in $D$. In particular, take the side 
lobe corresponding to the sequence element $x$ and its side lobe value $C(x)$. By the previous calculation

$$
C(x)= \begin{cases}v+4(\mu-k)-2, & \text { if } x \notin D, \\ v+4(\lambda-k)+2, & \text { if } x \in D .\end{cases}
$$

In the special case where $\mu-\lambda=1$, a single side lobe value will occur. Specifically, all side lobe values will have the single value given by $v+4(\mu-k)-2$. It has been shown by Theorem 2 that this condition only holds for when the partial difference set satisfies the Paley parameters or when $D$ is the sporadic example with parameters $(243,22,1,2)$.

Example 4 As an example of Theorem 4, take the famous Paley partial difference set of length 13 . This binary sequence is given by

$$
D=[-+-++----++-+]
$$

and its periodic correlation can be calculated to be

$$
C(D)=[13-3+-3-3++++-3-3+-3] .
$$

Using Theorem 3,

$$
S=[0+-++----++-+]
$$

whose periodic correlation is two-valued. This correlation is calculated to be

$$
C(S)=[12------------] .
$$

\subsection{Optimal high-energy ternary sequence construction (OHETS)}

Take the constructions from Theorems 3 and 4. Using the cases where the sequences have the Paley parameters, we call the resulting sequence, $S$, which is created by puncturing, an optimal high-energy ternary sequence (OHETS). Before stating and proving the main theorem, we present a few necessary lemmas for its proof.

Lemma 2 For a prime power $q \equiv 3(\bmod 4), S N^{(-1)}=\left(\frac{q+1}{4}\right) G-\left(\frac{q+1}{4}\right)-S$. 
Proof By Eq. (6), $S N^{(-1)}=S S$. Further, $S S=S(G-N-1)$ by Eq. (7) and $N=S^{(-1)}$ by Eq. (5). Now expand as follows:

$$
\begin{aligned}
S N^{(-1)} & =S S \\
& =S(G-N-1) \\
& =S G-S N-S \\
& =S G-S S^{(-1)}-S \\
& =\left(\frac{q-1}{2}\right) G-\left(\frac{q+1}{4}\right)-\left(\frac{q-3}{4}\right) G-S \\
& =\left(\frac{q+1}{4}\right) G-\left(\frac{q+1}{4}\right)-S .
\end{aligned}
$$

Lemma 3 For a prime power $q \equiv 3(\bmod 4), S^{(-1)} N=\left(\frac{q+1}{4}\right) G-\left(\frac{q+1}{4}\right)-N$.

Proof Since $S N^{(-1)}$ and $S^{(-1)} N$ are inverses of one another, it is sufficient to compute the inverse of both sides of the result from Lemma 2 . Note that the only change on the right side will be that $S$ inverts to $N$.

Lemma 4 For a prime power $q \equiv 1(\bmod 4), S N=\left(\frac{q-1}{4}\right) G-\left(\frac{q-1}{4}\right)$.

Proof By using a very similar method as in Lemma 2, except using the equations presented in Example 2, we find the following:

$$
\begin{aligned}
S N & =S(G-S-1) \\
& =S G-S S-S) \\
& =S G-S S^{(-1)}-S \\
& =\left(\frac{q-1}{2}\right) G-\left(\frac{q-1}{2}\right)-\left(\frac{q-5}{4}\right) S-\left(\frac{q-1}{4}\right) N-S \\
& =\left(\frac{q-1}{2}\right) G-\left(\frac{q-1}{2}\right)-\left(\frac{q-1}{4}\right)(S+N) \\
& =\left(\frac{q-1}{2}\right) G-\left(\frac{q-1}{2}\right)-\left(\frac{q-1}{4}\right)(G-1) \\
& =\left(\frac{q-1}{4}\right) G-\left(\frac{q-1}{4}\right) .
\end{aligned}
$$

Theorem 5 Let $q$ be an odd prime power. Let $G=(G F(q),+)$ where $S$ and $N$ denote the square and non-square elements of $G F(q) \backslash\{0\}$. Then, the ternary sequence (array) $\boldsymbol{a}=\left(a_{i}\right)$ indexed by $(G F(q),+)$ defined by: 


$$
a_{i}= \begin{cases}0, & \text { if } i=0 \\ +1, & \text { if } i \text { is in } S \\ -1, & \text { if } i \text { is in } N\end{cases}
$$

satisfies that $\boldsymbol{a a}^{(-1)}=(q-1)-S-N$ in the group ring $Z[G]$. That is to say that all side lobes of $\boldsymbol{a}$ are -1 and the main lobe is $q-1$.

Proof We break this into two cases. Case 1 is for $q \equiv 3(\bmod 4)$ and Case 2 is for $q \equiv 1(\bmod 4)$.

Case 1 Assume that $q \equiv 3(\bmod 4)$. Then, the sequence a $=S-N$ has an autocorrelation $\mathbf{a a}^{(-1)}$ computed by

$$
\begin{aligned}
\mathbf{a a}^{(-1)} & =(S-N)(S-N)^{(-1)} \\
& =S S^{(-1)}+N N^{(-1)}-S N^{(-1)}-N S^{(-1)} .
\end{aligned}
$$

Next, using Eq. (4) for the first two terms and Lemmas 2 and 3 for the last two terms we get

$$
\begin{aligned}
\mathbf{a a}^{(-1)} & =2\left[\left(\frac{q+1}{4}\right)+\left(\frac{q-3}{4}\right) G\right]-\left(\frac{q+1}{2}\right) G+\left(\frac{q+1}{2}\right)+(G-1) \\
& =\left(\frac{q+1}{2}\right)+\left(\frac{q-3}{2}\right) G-\left(\frac{q+1}{2}\right) G+\left(\frac{q+1}{2}\right)+(G-1) \\
& =\left(\frac{2 q+2+2 q+2-4}{4}\right)+\left(\frac{2 q-6-2 q-2+4}{4}\right) G \\
& =q-G .
\end{aligned}
$$

This finishes Case 1 .

Case 2 Assume that $q \equiv 1(\bmod 4)$. Then, the sequence $\mathbf{a}=S-N$ and we examine its autocorrelation $\mathbf{a a}^{(-1)}$ directly. Note that

$$
\begin{aligned}
\mathbf{a a}^{(-1)} & =(S-N)(S-N)^{(-1)} \\
& =S S^{(-1)}+N N^{(-1)}-S N^{(-1)}-N S^{(-1)} \\
& =S^{2}+N^{2}-2 S N
\end{aligned}
$$

by Eqs. (10) and (11). Now expanding by using the equations with Example 2 and Lemma 4:

$$
\begin{aligned}
\mathbf{a a}^{(-1)}= & S^{2}+N^{2}-2 S N \\
= & (q-1)+\left(\frac{q-5}{4}\right)(S+N)+\left(\frac{q-1}{4}\right)(S+N) \\
& -2\left[\left(\frac{q-1}{4}\right) G-\left(\frac{q-1}{4}\right)\right]
\end{aligned}
$$


Table 1 The first ten OHETS

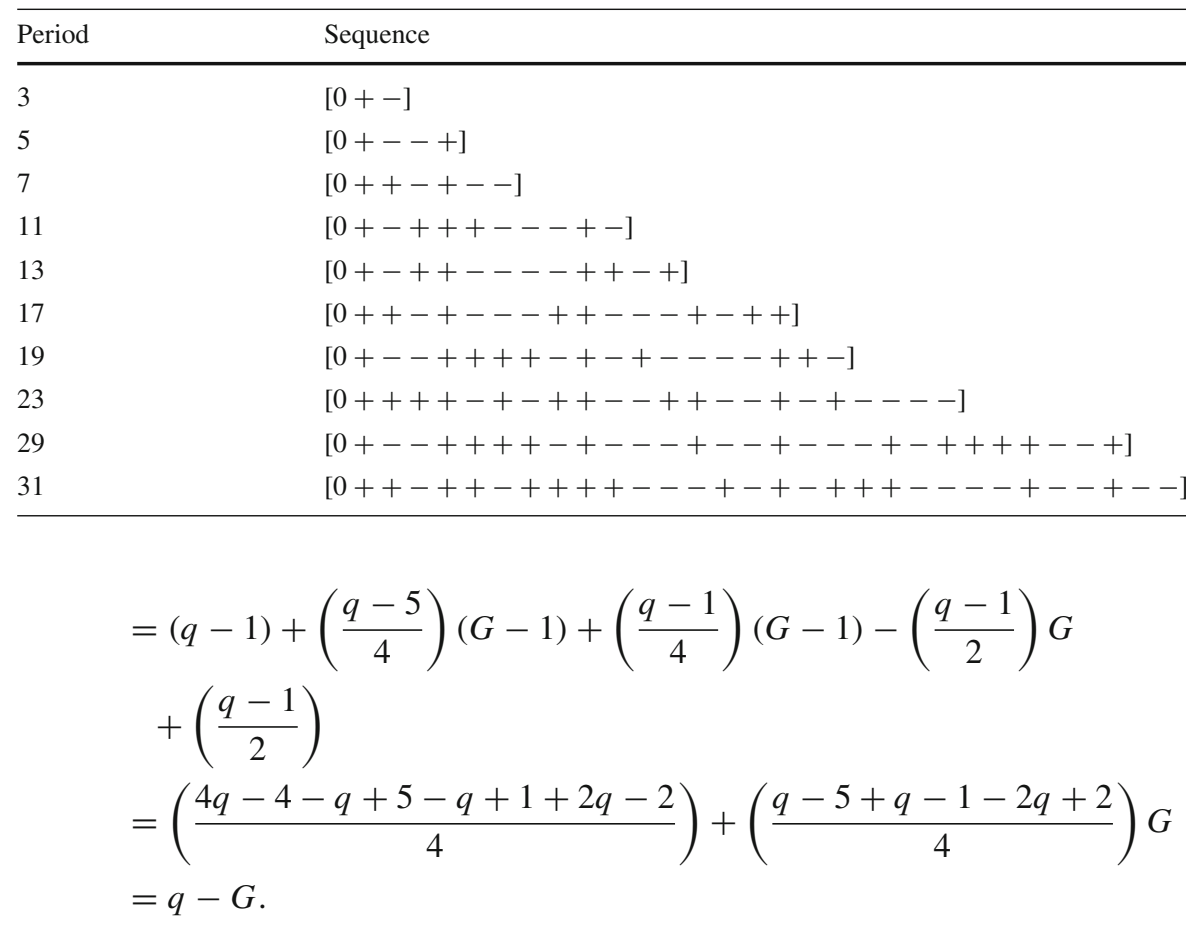

Remark The case for $q \equiv 1(\bmod 4)$ above provides optimal sequences. That is to say that these sequences provide the minimum possible sum of squares in the coefficients of their ACF. This is important as the Paley sequences for $q \equiv 3(\bmod 4)($ See Example 1$)$ give ACF coefficients of -1 and the case for $q \equiv 1(\bmod 4)($ See Example 2$)$ give $1 \mathrm{~s}$ and $-3 \mathrm{~s}$. Thus, our sequences provide a better (smaller) sum of squares for the $q \equiv 1(\bmod 4)$ case.

Proof For the OHETSs above, we assume prime power period $q$ with a single punctured position at $i=0$. Thus, the dot product of the original sequence a with any of its translates will result in exactly two entries being multiplied by the 0 . This implies exactly two values of the dot product will cancel out, leaving $q-2$ terms each of which is \pm 1 . This means that an odd number of $\pm 1 \mathrm{~s}$ are being summed and the final value can not be even (i.e., can not be 0 ). Therefore, these new sequences provide the optimal value for the sum of squares since $( \pm 1)^{2}$ is the smallest odd integer.

\subsubsection{List of optimal high-energy ternary sequences and arrays}

In Table 1, we show the OHETS sequences for the first lengths up to $N=31$. Note that these ternary sequences are clearly high in energy.

These OHETS sequences have optimal periodic autocorrelation functions, as we proved; however, their aperiodic autocorrelation functions, as in Definition 7, are not 
Table 2 Aperiodic autocorrelations of the first ten OHETS (using the shift with highest GMF)

\begin{tabular}{|c|c|}
\hline Period & Aperiodic autocorrelation \\
\hline 3 & {$\left[\begin{array}{lll}2 & -1 & 0\end{array}\right]$} \\
\hline 5 & {$\left[\begin{array}{lllll}4 & 0 & 0 & -1 & -1\end{array}\right]$} \\
\hline 7 & 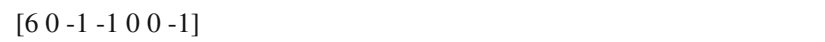 \\
\hline 11 & 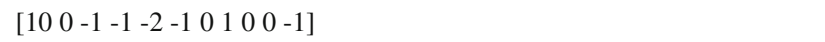 \\
\hline 13 & 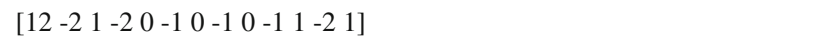 \\
\hline 17 & {$\left[\begin{array}{llllllllllllllllllll}16 & 0 & -1 & 0 & -1 & 1 & 2 & -1 & 0 & -1 & 0 & -3 & -2 & 0 & -1 & 0 & -1\end{array}\right]$} \\
\hline 19 & 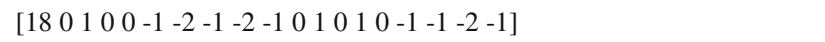 \\
\hline 23 & 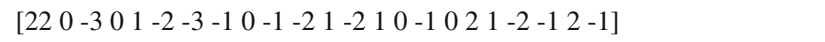 \\
\hline 29 & 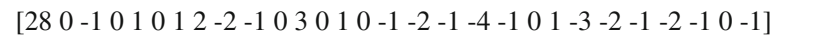 \\
\hline 31 & 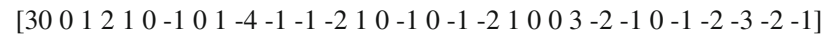 \\
\hline
\end{tabular}

Table 3 First two optimal high-energy arrays

\begin{tabular}{ll}
\hline Period & Array \\
\hline $3 \times 3$ & {$\left[\begin{array}{l}0-- \\
++- \\
+-+\end{array}\right]$} \\
$5 \times 5$ & {$\left[\begin{array}{l}0---- \\
++-+- \\
+++-- \\
+--++ \\
+-+-+\end{array}\right]$}
\end{tabular}

optimal. We present them in Table 2. For the aperiodic autocorrelation function, we present the one associated with the shift, of the original sequence in Table 1, providing the largest Golay merit factor.

In Table 3, we show the first two OHETS-based arrays whose periodic autocorrelation functions are again optimal.

\subsubsection{Generation of conference matrices with group developed core}

A conference matrix, $C$, is a square ternary sequence with exactly one zero entry per row (column) satisfying $C C^{T}=k I$ for some constant $k$ and identity matrix $I$. These objects are known not to exist in general if they are cyclically developed; however, we can generate an almost-cyclically developed conference matrix using our OHETS sequences.

Theorem 6 A conference matrix, $C$, of size $q+1$, where $q$ is an odd prime, exists where its border consists of all $+1 s$ and the core (non-border entries) is a cyclically developed matrix from an OHETS.

Proof Let a be any OHETS of length $q$. Define $A$ as the matrix consisting of the sequence a and all of its cyclic shifts. Thus, $A$ is a cyclically developed square matrix 
Fig. 1 Construction of conference matrix with group developed core

$$
C=\left[\begin{array}{ccc}
0 & +\cdots & + \\
+ & \\
\vdots & A \\
+ &
\end{array}\right]
$$

of size $q \times q$. Define a new matrix $C$ by taking $A$ and bordering it on the left and the top by a column and row of $+1 \mathrm{~s}$ and let $C_{1,1}=0$. $C$ can be visualized as in Fig. 1 . The matrix $C$ has the property that $C C^{T}=q I_{q+1}$ where $I_{n}$ is the identity matrix of size $n$. This can be easily verified by looking at the dot product of any two rows of $C$. Let $i$ and $j$ represent the row and column indices of $C$, respectively.

Case $1(i=j)$ It is clear when any row of $C$ is dotted with itself that the answer is $q$. This is trivially true for the first row of $C$ by construction. For all other rows, by definition $A$ has $q-1$ nonzero entries and one more is added to the left. Thus, any row dotted with itself is $q$.

Case $2(i \neq 1, j \neq 1)$ For any two rows, both of which are not the first of $C$, there is exactly one extra copy of +1 to the dot product. Since all other terms of the product come from a OHETS and its shift, then their dot product is -1 by construction. Then, $+1+-1=0$ is the dot product of any two such rows.

Case 3 ( $i=1$ and $j>1$ ) If the first row is involved, then note the 0 at the beginning means the dot product will only consist of the sum of entries in the original OHETS. Thus, the product will simply be the sum of terms in the sequence a. By construction, these OHETS are balanced when looking at the number of $\pm 1 s$, and thus, the dot product is again 0 .

This proves that $C C^{T}=q I_{q+1}$. Further, it is a conference matrix as each row contains exactly one 0 element.

Example 5 Using the data provided in Table 1, we can construct such a conference matrix of size 6 . Let $\mathbf{a}=[0+--+]$. Then, we can "develop" $A$ cyclically so that

$$
A=\left[\begin{array}{c}
0+--+ \\
+0+-- \\
-+0+- \\
--+0+ \\
+--+0
\end{array}\right]
$$

and then construct $C$ by

$$
C=\left[\begin{array}{c}
0+++++ \\
+0+--+ \\
++0+-- \\
+-+0+- \\
+--+0+ \\
++--+0
\end{array}\right]
$$

It can be verified that $C C^{T}=5 I_{6}$. 


\subsection{High-energy binary peak shifting sequence construction (HEBPSS)}

Using the properties of the perfect squares in the field $G F(q)$ as above, we prove the existence of another infinite family of sequences which reside in the group $H=$ $Z_{2} \times G$. Here, we have $q \equiv 1(\bmod 4)$ is a prime and $G=G F((q),+)$. This family has the property that their main lobe is of size $q$, with exactly two side lobes of size $\frac{3(q-1)}{4}$ and all remaining side lobes of $\frac{q-1}{2}$. Further, these sequences are flexible in that the position of the $\frac{3(q-1)}{4}$ side lobe pair can be moved around, in some sense. Thus, the term "peak shifting." We first show the proof for this family of binary sequences. In the following theorem, we use that for a subset $S$ of $G$, define $S\left(x^{j}\right)$ to be the resulting set in $Z_{2 q}$ after embedding $G=Z_{q}$ in $Z_{2 q}$ and applying the function $x \rightarrow x^{j}$ to the elements of $S$.

Theorem 7 Let $q \equiv 1(\bmod 4)$ be a prime and let $P$ be the set of all nonzero squares in $G F(q)-\{0\}$. Then, working in the group $Z_{2 q}$, we define the binary sequence given by $\boldsymbol{b}=P\left(x^{2}\right)\left(1+x^{2 k+1}\right)+x^{2 k+1}$, of period $2 q$, where we fix a value $k \in$ $\left\{0,1,2, \ldots, \frac{q-1}{2}\right\}$. This sequence has a periodic autocorrelation function with the main lobe of $q$, exactly two side lobes of size $\frac{3(q-1)}{4}$ positioned at the entries $x^{2 k+1}$ and $x^{-2 k-1}$, and all other side lobes have a value of $\frac{q-1}{2}$. In the special case where $k=\frac{q-1}{2}$, then all side lobes have a value of $\frac{q-1}{2}$ except the midpoint which will take on the value $q-1$.

Proof Let $q$ be a prime where $q \equiv 1(\bmod 4)$ and assume that $P$ is the set of all nonzero squares in $Z_{q}$. Let $\mathbf{b}=P\left(x^{2}\right)(1+y)+y$, where $y=x^{2 k+1}$ to make the following proof easier to read. We proceed by computing the autocorrelation function bb $^{(-1)}$ directly and noting that $P\left(x^{2}\right)=P^{(-1)}\left(x^{2}\right)$ by Eq. (10).

$$
\begin{aligned}
\mathbf{b b}^{(-1)} & =\left[P\left(x^{2}\right)(1+y)+y\right]\left[P^{(-1)}\left(x^{2}\right)\left(1+y^{-1}\right)+y^{-1}\right] \\
& =P\left(x^{2}\right) P\left(x^{2}\right)\left(2+y+y^{-1}\right)+P\left(x^{2}\right)\left(2+y+y^{-1}\right)+1 \\
& =\left[P\left(x^{2}\right) P\left(x^{2}\right)+P\left(x^{2}\right)\right]\left[2+y+y^{-1}\right]+1 .
\end{aligned}
$$

Now, use Eq. (8) for the expansion of $P\left(x^{2}\right) P\left(x^{2}\right)$ :

$$
\begin{aligned}
\mathbf{b b}^{(-1)}= & {\left[P\left(x^{2}\right) P\left(x^{2}\right)+P\left(x^{2}\right)\right]\left[2+y+y^{-1}\right]+1 } \\
= & {\left[\left[\frac{q-1}{2}+\frac{q-5}{4} P\left(x^{2}\right)+\frac{q-1}{4} N\left(x^{2}\right)\right]+P\left(x^{2}\right)\right]\left[2+y+y^{-1}\right]+1 } \\
= & q+\frac{q-1}{2} y+P\left(x^{2}\right)\left(\frac{q-1}{2}+\frac{q-1}{4} y+\frac{q-1}{4} y^{-1}\right) \\
& +N\left(x^{2}\right)\left(\frac{q-1}{2}+\frac{q-1}{4} y+\frac{q-1}{4} y^{-1}\right)+\frac{q-1}{2} y^{-1} \\
= & q+\frac{q-1}{2} y+\frac{q-1}{2}\left(P\left(x^{2}\right)+N\left(x^{2}\right)\right)+\frac{q-1}{4}\left(P\left(x^{2}\right)\right. \\
& \left.+N\left(x^{2}\right)\right)\left(y+y^{-1}\right)+\frac{q-1}{2} y^{-1} .
\end{aligned}
$$


At this point, note that the term $P\left(x^{2}\right)+N\left(x^{2}\right)$ contains every nonzero even power of $x$ exactly once. Next we examine the fourth part of the expansion above, whose coefficient is $\frac{q-1}{4}$ separately. We will prove that $\left(P\left(x^{2}\right)+N\left(x^{2}\right)\right)\left(y+y^{-1}\right)$ contains $y$ and $y^{-1}$ exactly once each and all other odd powers of $x$ exactly twice. First, by Eq. (12), we have that $G=P+N+1$ in the group $Z_{q}$. Thus, in the group $Z_{2 q}$, we have that $G\left(x^{2}\right)=P\left(x^{2}\right)+N\left(x^{2}\right)+1$. This allows us to write:

$$
\begin{aligned}
\left(P\left(x^{2}\right)+N\left(x^{2}\right)\right)\left(y+y^{-1}\right) & =\left(P\left(x^{2}\right)+G\left(x^{2}\right)-P\left(x^{2}\right)-1\right)\left(y+y^{-1}\right) \\
& =\left(G\left(x^{2}\right)-1\right)\left(y+y^{-1}\right) \\
& =G\left(x^{2}\right) y+G\left(x^{2}\right) y^{-1}-y-y^{-1} .
\end{aligned}
$$

Since $G\left(x^{2}\right)$ clearly contains all even powers of $x$, then $G\left(x^{2}\right) y$ and $G\left(x^{2}\right) y^{-1}$ must contain all odd powers of $x$ each. Recall that $y=x^{2 k+1}$ is itself an odd power of $x$. Thus,

$$
G\left(x^{2}\right) y+G\left(x^{2}\right) y^{-1}-y-y^{-1}=y+y^{-1}+2 \sum_{m \neq k} x^{2 m+1} .
$$

We can now substitute Eq. (15) into Eq. (13) yielding

$$
\begin{aligned}
\mathbf{b b}^{(-1)}= & q+\frac{q-1}{2} y+\frac{q-1}{2}\left(P\left(x^{2}\right)+N\left(x^{2}\right)\right)+\frac{q-1}{4}\left(P\left(x^{2}\right)\right. \\
& \left.+N\left(x^{2}\right)\right)\left(y+y^{-1}\right)+\frac{q-1}{2} y^{-1} \\
= & q+\frac{q-1}{2} y+\frac{q-1}{2}\left(P\left(x^{2}\right)+N\left(x^{2}\right)\right) \\
& +\frac{q-1}{4}\left(y+y^{-1}+2 \sum_{m \neq k} x^{2 m+1}\right)+\frac{q-1}{2} y^{-1} \\
= & q+\frac{3(q-1)}{4} y+\frac{q-1}{2}\left(P\left(x^{2}\right)+N\left(x^{2}\right)\right) \\
& +\frac{q-1}{2}\left(\sum_{m \neq k} x^{2 m+1}\right)+\frac{3(q-1)}{4} y^{-1} .
\end{aligned}
$$

This shows the sequence $\mathbf{b}$ has the main lobe $q$, all side lobes are $\frac{q-1}{2}$ except exactly the side lobe pair $y$ and $y^{-1}$ whose values are $\frac{3(q-1)}{4}$. Finally, look at the special case where $k=\frac{q-1}{2}$. This implies that $y=y^{-1}$ as $y=x^{2 k+1}=x^{q}=x^{-q}=y^{-1}$ where powers are computed modulo $2 q$. This results in a change to Eq. (14) making

$$
\begin{aligned}
\left(P\left(x^{2}\right)+N\left(x^{2}\right)\right)\left(y+y^{-1}\right) & =\left(P\left(x^{2}\right)+G\left(x^{2}\right)-P\left(x^{2}\right)-1\right)\left(y+y^{-1}\right) \\
& =\left(G\left(x^{2}\right)-1\right)(y+y) \\
& =2 y\left(G\left(x^{2}\right)-1\right) .
\end{aligned}
$$


When Eq. (16) is substituted into Eq. (13), we now get

$$
\begin{aligned}
\mathbf{b b}^{(-1)}= & q+\frac{q-1}{2} y+\frac{q-1}{2}\left(P\left(x^{2}\right)+N\left(x^{2}\right)\right)+\frac{q-1}{4}\left(2 y\left(G\left(x^{2}\right)-1\right)\right) \\
& +\frac{q-1}{2} y \\
= & q+\frac{q-1}{2} y+\frac{q-1}{2}\left(P\left(x^{2}\right)+N\left(x^{2}\right)\right)+\frac{q-1}{2}\left(y\left(G\left(x^{2}\right)-1\right)\right) \\
& +\frac{q-1}{2} y \\
= & q+(q-1) y+\frac{q-1}{2}\left[\left(P\left(x^{2}\right)+N\left(x^{2}\right)+\left(y\left(G\left(x^{2}\right)-1\right)\right)\right] .\right.
\end{aligned}
$$

This calculation shows the main lobe of size $q$, all side lobes (excluding $y$ ) of size $\frac{q-1}{2}$, and a single peak at the midpoint $y$ of size $q-1$.

The previous construction works for sequences, but generalizes nicely to the more general domain of arrays which we show next. It is our hope that with the rapid advancement of technology that engineering applications that employ sequences could easily be adapted using their higher-dimensional "array" counterparts instead; the advantage being "arrays" embrace a much larger spectrum, in comparison with their "sequence" compeers.

Theorem 8 Let $H=Z_{2} \times G$ be the group where $Z_{2}=<t>$ and $G$ is the additive group of the finite field $G F(q)$ where $q \equiv 1(\bmod 4)$ is a prime power. Let $g$ be any element of $G$ and $P$ represent the set of all nonzero squares in $G F(q)$. Then, the group ring object, in $Z[H]$ where we write the group $H$ multiplicatively, $W=P(1+t g)+t g$ satisfies that its periodic autocorrelation has the main lobe of size q, side lobes of size $\frac{q-1}{2}$ and exactly two side lobes represented by the elements $t g$ and $\mathrm{tg}^{-1}$ of size $\frac{3(q-1)}{4}$. If $g=g^{-1}$ then the two larger side lobes merge together with size $q-1$ at $t$ because $g=g^{-1}$ implies $g=1$ since $|G|$ is odd and hence $t g=t$.

Proof Compute the autocorrelation function directly using virtually identical arguments as in Theorem 7. We note that $g G=G$ and $G+t G=H$.

$$
\begin{aligned}
W W^{(-1)} & =(P(1+\operatorname{tg})+\operatorname{tg})\left(P\left(1+t g^{-1}\right)+t g^{-1}\right) \\
& =\left[\left(\frac{q-1}{2}\right)+\left(\frac{q-1}{4}\right)(G-1)\right]\left[2+t g+t g^{-1}\right]+1 \\
& =\left(\frac{q+1}{2}\right)+\left(\frac{q-1}{2}\right) H+\left(\frac{q-1}{4}\right)\left(t g+t g^{-1}\right)
\end{aligned}
$$

which shows that the main lobe has a value of $q$, every side lobe that is not represented by $t g$ or $t g^{-1}$ has a value of $\frac{q-1}{2}$ and the two side lobes represented by $t g$ and $t g^{-1}$ have a value of $\frac{3(q-1)}{4}$ and in the case where $t g=\mathrm{tg}^{-1}=t$ we get a single side lobe with value $q-1$. Thus, $W$ is the array we set out to describe. 
Table 4 List of first five HEBPSSs with $k=0$

\begin{tabular}{ll}
\hline$q($ Period $=2 q)$ & Sequence \\
\hline 5 & {$[0+++00000++]$} \\
13 & {$[0+++00++++000000000++++00++]$} \\
17 & {$[0+++++00++0000000++++0000000++00++++]$} \\
29 & {$[0+++0000++++++++00++0000000++0$} \\
& $000++0000000++00++++++++00000++]$ \\
37 & {$[0+++00++++0000++00++++++++0000000++000$} \\
& $00000++0000000++++++++00++00000++++00++]$
\end{tabular}

Table 5 List of all peak shifts of HEBPSSs with period $2 q=10$

$\left.\begin{array}{lll}\hline k & \text { Sequence } & \text { Periodic autocorrelation } \\ \hline 0 & {[0+++0} & 0\end{array}\right]$

Table 6 First two high-energy binary peak shifting arrays

\begin{tabular}{|c|c|}
\hline Period & Array \\
\hline $3 \times 6$ & {$\left[\begin{array}{cccccc}0 & + & + & + & + & + \\
0 & 0 & 0 & 0 & + & + \\
0 & 0 & + & + & 0 & 0\end{array}\right]$} \\
\hline $5 \times 10$ & 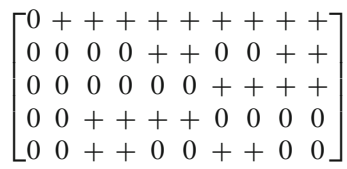 \\
\hline
\end{tabular}

\subsubsection{List of high-energy binary peak shifting sequences and arrays}

In Table 4, we show the first five HEBPS sequences explicitly as binary, $\{0,1\}$, objects.

To demonstrate the "peak shifting" capability of this construction, in Table 5, we examine the three distinct shifts of the length 10 HEBPSS. Note the translation of the periodic autocorrelation side lobe of value 3 as it approaches the midpoint.

Lastly, we show in Table 6 the first two binary HEBPS arrays.

\subsubsection{Conversion from binary $\{0,1\}$ to $\{-1,1\}$ sequences (arrays)}

The sequences given in Theorem 7 are binary objects and have elements $\{0,1\}$. It is sometimes useful to convert these $\{0,1\}$ objects to $\{-1,1\}$ objects. They are essentially the same sequences where all 0 s have been replaced with $-1 \mathrm{~s}$. The following proposition gives a connection between the periodic side lobes of binary sequences which have been converted from a $\{0,1\}$ to $\{-1,1\}$ sequence. 
Proposition 3 A binary $\{0,1\}$ sequence a whose periodic autocorrelation function is given by $\boldsymbol{a a}^{(-1)}=\sum \lambda_{g} g$ has a corresponding binary $\{-1,1\}$ autocorrelation function $\tilde{\boldsymbol{a}} \tilde{\boldsymbol{a}}^{(-1)}=\sum \mu_{g} g$ where

$$
\mu_{g}=|G|-4\left(\chi_{0}(\boldsymbol{a})-\lambda_{g}\right) .
$$

In this formula, $|G|$ represents the order of the group $G$ and $\chi_{0}$ is the principal character of the group $G$.

Proof Let $\mathbf{a}=\sum a_{i} g_{i}$ be a $(0,1)$ sequence where $g_{i}$ represents the elements of a group $G$. Define $P=\left\{g_{i} \mid a_{i}=1\right\}$ and $N=\left\{g_{i} \mid a_{i}=0\right\}$. Note that $\mathbf{a}=P$. Further, let the autocorrelation function of a be given by $\mathbf{a} \mathbf{a}^{(-1)}=\sum \lambda_{g} g$. Now, define a new sequence $\tilde{\mathbf{a}}=P-N$ and label its autocorrelation function $\tilde{\mathbf{a}} \tilde{\mathbf{a}}^{(-1)}=\sum \mu_{g}$. Now we compute ãã $\tilde{a}^{(-1)}$ :

$$
\begin{aligned}
\tilde{\mathbf{a a}}^{(-1)} & =\sum \mu_{g} g \\
& =(2 P-G)(2 P-G)^{(-1)} \\
& =(2 P-G)\left(2 P^{(-1)}-G\right) \\
& =4 P P^{(-1)}-2 P G-2 P^{(-1)} G+G^{2} \\
& =4 \mathbf{a a}^{(-1)}-2|P| G-2|P| G+|G| G \\
& =4 \mathbf{a a}^{(-1)}-4|P| G+|G| G \\
& =|G| G-4\left(|P| G-\mathbf{a a}^{(-1)}\right) \\
& =|G| G-4\left(\chi_{0}(\mathbf{a})-\sum \lambda_{g} g\right) .
\end{aligned}
$$

This shows that

$$
\sum \mu_{g} g=|G| G-4\left(\chi_{0}(\mathbf{a}) G-\sum \lambda_{g} g\right)
$$

We finish by fixing an element $g^{*} \in G$.

$$
\mu_{g^{*}} g^{*}=|G| g^{*}-4\left(\chi_{0}(\mathbf{a}) g^{*}-\lambda_{g^{*}} g^{*}\right)
$$

which shows that the correlations are related to one another by $\mu_{g^{*}}=|G|-$ $4\left(\chi_{0}(\mathbf{a})-\lambda_{g^{*}}\right)$ as claimed.

We finish this section by writing the sequences in Tables 4, 5, and 6 as binary $\{-1,1\}$ objects . 
Table 7 List of first five HEBPSSs with $k=0$

\begin{tabular}{ll}
\hline$q($ Period $=2 q)$ & Sequence \\
\hline 5 & {$[-+++----++]$} \\
13 & {$[-+++--++++------++++--++]$} \\
17 & {$[-+++++--++-----+++++-----++--++++]$} \\
29 & {$[-+++----++++++++--++------++-$} \\
& $---++-----+++-++++++++----++]$ \\
37 & {$[-+++--++++----++--++++++++-----++---$} \\
& $-----++-----+++++++++--++----++++--++]$ \\
\hline
\end{tabular}

Table 8 List of all peak shifts of HEBPSSs with period $2 q=10$

\begin{tabular}{lll}
\hline$k$ & Sequence & Periodic autocorrelation \\
\hline 0 & {$[-+++----++]$} & {$[10+2-2-2-2-2-2-2-2+2]$} \\
1 & {$[-+++-+--+-]$} & {$[10-2-2+2-2-2-2+2-2-2]$} \\
2 & {$[--++-+-++-]$} & {$[10-2-2-2-2+6-2-2-2-2]$} \\
\hline
\end{tabular}

Table 9 First two high-energy binary peak shifting arrays

\begin{tabular}{ll}
\hline Period & Array \\
\hline $3 \times 6$ & {$\left[\begin{array}{l}-+++++ \\
----++ \\
--++--\end{array}\right]$} \\
$5 \times 10$ & {$\left[\begin{array}{l}-+++++++++ \\
----++--++ \\
-----++++ \\
--++++---- \\
--++--++-\end{array}\right]$}
\end{tabular}

\section{Sequences and their performance measures}

In this section, we provide our new sequences given above, as well as other known sequences, and present them with computational properties. The properties we provide are: length, energy, energy efficiency, Golay merit factor, peak-to-max side lobe ratio, peak-to-average side lobe ratio, peak-to-neighbor side lobe ratio, spectral peak-toaverage ratio, and Doppler tolerance. Many of these properties require the so-called aperiodic correlation Function to be calculated for a given sequence. We define this first (Tables 7, 8 and 9).

Definition 7 The aperiodic cross-correlation function of two sequences $\mathbf{a}$ and $\mathbf{b}, C_{\text {aper }}$, is defined by

$$
C_{\text {aper }}(l)=\sum_{i=0}^{n-1-l} a_{i} b_{i+l}^{*}
$$


where $a_{i}^{*}$ represents the complex conjugate of $a_{i}$ and $0 \leq l<n$. If $\mathbf{a}=\mathbf{b}$, then this is referred to as the aperiodic autocorrelation function.

Definition 8 The Golay merit factor (GMF) of a sequence $\mathbf{a}$ is defined by

$$
\mathrm{GMF}=\frac{C_{\mathrm{aper}}^{2}(0)}{2 \sum_{l=1}^{n-1} C_{\mathrm{aper}}^{2}(l)} .
$$

Definition 9 The peak-to-max side lobe ratio (PMSR) of a sequence is defined to be

$$
\operatorname{PMSR}=\frac{C_{\mathrm{aper}}^{2}(0)}{\max \left\{C_{\mathrm{aper}}^{2}(m)\right\}}
$$

where $m \neq 0$.

Definition 10 The peak-to-average side lobe ratio (PASR) of a sequence is defined to be

$$
\operatorname{PASR}=\frac{C_{\mathrm{aper}}^{2}(0)}{\operatorname{mean}\left\{C_{\mathrm{aper}}^{2}(m)\right\}}
$$

Definition 11 The peak-to-neighbor side lobe ratio (PNSR) of a sequence is defined to be

$$
\operatorname{PNSR}=\frac{C_{\text {aper }}(0)}{C_{\text {aper }}^{2}(1)}
$$

Definition 12 The spectral peak-to-average ratio (SPAR), as described in [9], is calculated in the following way: First we pad the sequence a with zeros on both sides. Call this new sequence $\mathbf{b}=[0 \mathbf{a} 0]$. The number of zeros, on both sides, is equal to the sequence length itself. Second, create the vector $F=|f f t(\mathbf{b})|^{2}$ where $f f t$ is the fast Fourier transform function in MATLAB. Finally, the SPAR can be found by:

$$
\operatorname{SPAR}=\frac{\max \{F\}}{\operatorname{mean}\{\hat{F}\}}
$$

where $\hat{F}=F \backslash \max \{F\}$.

Definition 13 The correlation energy of a sequence is defined as

$$
\mathrm{CE}=\sum_{l=1}^{n-1} C^{2}(l) .
$$


Definition 14 The periodic Golay merit factor (pGMF) of a sequence a is defined by

$$
\mathrm{pGMF}=\frac{C^{2}(0)}{\sum_{l=1}^{n-1} C^{2}(l)} .
$$

\subsection{The performance measures of selected sequences}

The data in Table 10 present all of the relevant properties mentioned above for selected sequences. Note that since the aperiodic correlation function of a function is dependent on the sequence's shift, of which there are $n$, these values have been calculated using the shift providing the largest GMF. From this table, we seek to examine sequences which achieve large $E, E_{\text {eff }}$, GMF, PMSR, PASR, PNSR, and pGMF. We seek all other values to be minimized.

From the sequences examined, we can see that our OHETS sequences provide a good balance between the sequence's energy and its Golay merit factor. In particular, when comparing the OHETS to the Paley it will frequently improve the GMF-never losing much if any. Another big advantage comes from our correlation energy and periodic GMF when examining the Paley sequence to the OHETS sequence of the same length. This is especially true for lengths which are congruent to $1(\bmod 4)$ where we see large improvements in performance.

\section{Sets of sequences with good properties}

\subsection{McLaughlin's algorithm for generating good sets of sequences}

In this section, we take a look at an algorithm proposed by McLaughlin [9]. Given a set of sequences, it is good for applications if this set has an overall large average GMF, small average SPAR, and small average pairwise aperiodic cross-correlation. Ideally, each sequence should have perfect periodic autocorrelations meaning the periodic side lobes are all zero. McLaughlin's patent suggests to start with a set of $m$ perfect sequences which are all inequivalent in the sense that no sequence in the set can be shifted to be another. For each of these $m$ sequences, he looks for the $w$ shifts which give the smallest values of $\frac{\mathrm{SPAR}}{\mathrm{GMF}}$. The number of shifts, $w$, which are chosen for each sequence can be made as large or small as wanted though choosing $w>2$ greatly slows the computational process. Once the best $w$ shifts for each sequence are found, he computes all possible $w^{m}$ (aperiodic) cross-correlations and in the end chooses the set with the smallest average cross-correlation. It is important to note here that we were not able to match his values for SPAR exactly, and thus, we proceed by taking his starting set and using our SPAR program to generate our own final set of sequences. This algorithm can be summarized by the following steps:

1. Obtain a set of sequences which are pairwise inequivalent by shifting.

2. For each sequence, find the $w$ shifts with smallest $\frac{\text { SPAR }}{\text { GMF }}$.

3. Generate all possible sets of sequences by using the shifts found in step 2 .

4. For each set, compute all possible pairwise cross-correlations. 


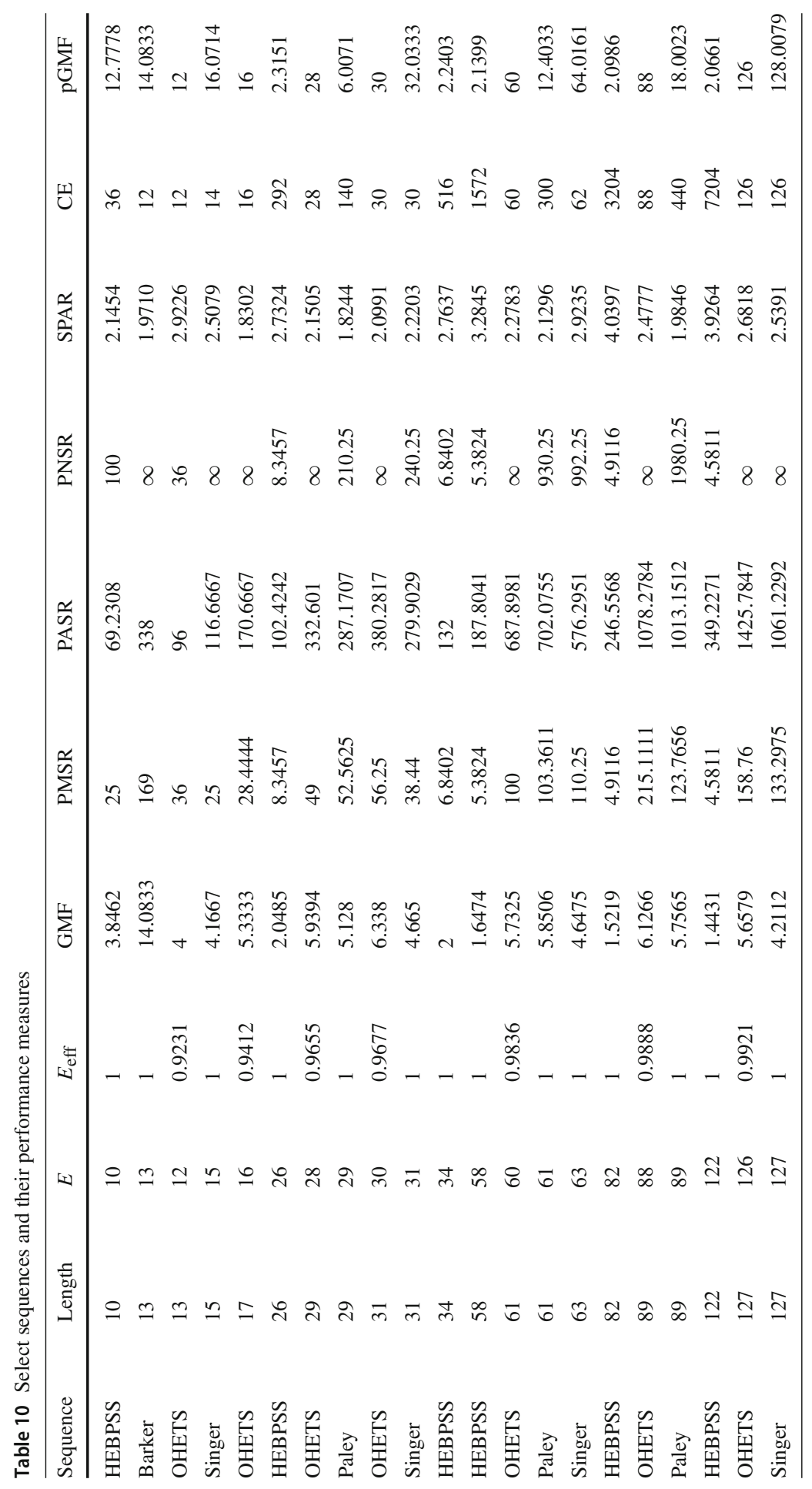


5. Compare the average cross-correlations and accept the set with smallest average cross-correlation as the final, improved, set.

We start by looking at one example set given by McLaughlin: Below is McLaughlin's starting set of 20 perfect periodic ternary sequences with the main lobe 25 (Tables 11, 12 and 13).

$$
\begin{aligned}
& --++-++++-+0+++0-0-+0--+-++00+- \\
& -00--+-++0+-0+0+++0--+++-+++-+- \\
& -+-+-0+00++--0-+++++-+++-0++-0- \\
& -+-+-++--+0+---0+0+++++0-+00++- \\
& -+++-0+00+-++0+-+-+++++--0--+0- \\
& -+++-++00+-0+0++-+++0----+-+0+- \\
& 0---0+-++-0+-+-+++--+++00+0+++- \\
& 0-++0-+++-+++++-0--++00+0-+-+-- \\
& 0+--0--+++++-+-+0++-+00+0-+++-- \\
& 0++-0+-+++0-++++-++----00+0+-+- \\
& +--++++++-0+-+-0-0-++--0++00++- \\
& +-+0+00----++-++++-0+++-+0-++0- \\
& +-+++-+++--0+++0+0-+0++-+--00-- \\
& +0-++++--0-+---+0+0++00-++-+++- \\
& +00++-+--0+-0-0+++0+-++++-++--- \\
& +0+-+----0+++-++0+0-+00++-+++-- \\
& ++00+-0+++++0+0---+0+--++-+-+-- \\
& ++00++0--++-0-0-+-+0-++++++--+- \\
& +++-++-00++0+0+---+0--++++-0+- \\
& +++0+00+++--+++-+-+0-++-+0---0-
\end{aligned}
$$

McLaughlin's ending set of 20 perfect ternary sequences where $w=1$ after processing with our program.

$$
\begin{aligned}
& -+0--+-++00+---++-++++-+0+++0-0 \\
& +-++0+-0+0+++0--+++-+++-+--00-- \\
& ++--0-+++++-+++-0++-0--+-+-0+00 \\
& ---0+0+++++0-+00++--+-+-++--+0+ \\
& ++++--0--+0--+++-0+00+-++0+-+-+ \\
& +00+-0+0++-+++0----+-+0+--+++-+ \\
& -+++--+++00+0+++-0---0+-++-0+-+ \\
& 00+0-+-+--0-++0-+++-+++++-0--++ \\
& +-+-+0++-+00+0-+++--0+--0--++++ \\
& -00+0+-+-0++-0+-+++0-++++-++--- \\
& ++--0++00++-+--++++++-0+-+-0-0- \\
& ---++-++++-0+++-+0-++0-+-+0+00-
\end{aligned}
$$


Table 11 McLaughlin starting versus ending set (perfect ternary sequences, period 31, main lobe 25)

\begin{tabular}{llc}
\hline Measure & Starting set & Ending set \\
\hline Average GMF & 2.8169 & 5.3937 \\
Average SPAR & 3.0346 & 2.1086 \\
Average aperiodic cross-correlation & 2.4806 & 2.3919 \\
\hline
\end{tabular}

$$
\begin{aligned}
& --00--+-+++-+++--0+++0+0-+0++-+ \\
& 0-++-+++-+0-++++--0-+---+0+0++0 \\
& -0+++0+-++++-++---+00++-+--0+-0 \\
& +-+++--+0+-+----0+++-++0+0-+00+ \\
& +0+--++-+-+--++00+-0+++++0+0--- \\
& -0-0-+-+0-++++++--+-++00++0--++ \\
& ++0+0+---+-0--++++-0+-+++-++-00 \\
& +-+0-++-+0---0-+++0+00+++--+++-
\end{aligned}
$$

Next, we wish to compare a set of our OHETSs to see if we can match, or beat, the McLaughlin ending set shown above. We create our starting set by selecting our OHETS of period 31 from the construction in Theorem 5. This sequence is given by

$$
[0++-++-++++---+-+-+++----+--+--] .
$$

We then add to this single sequence another which was found by computer search. The second sequence, given by

$$
[0+++-++---++++-+---+-+--++++-++]
$$

has the property that all of its periodic side lobes are either 1 or -1 (the previous sequence has periodic side lobes which are all -1 ). We generate our starting set by computing all possible combinations of shifts, negations, and automorphisms of the two sequences and reducing the resulting list by removal of any sequences which are cyclic shifts of another in the list. The definitions for these operations are as follows:

Definition 15 The (cyclic) shift by $n$ places of a sequence a of period $N$, written as the group ring object $A=\sum_{m=0}^{N-1} a_{m} x^{m}$ where $G=<x>$, is

$$
A x^{n}=\sum_{m=0}^{N-1} a_{m} x^{m+n}
$$

for some integer $n$. 
Definition 16 The negation of a sequence a of period $N$, written as the group ring object $A=\sum_{m=0}^{N-1} a_{m} x^{m}$ where $G=<x>$, is

$$
-A=\sum_{m=0}^{N-1}-a_{m} x^{m} .
$$

Definition 17 An automorphism of a sequence a of period $N$, written as the group ring object $A=\sum_{m=0}^{N-1} a_{m} x^{m}$ where $G=<x>$, is

$$
A\left(x^{n}\right)=\sum_{m=0}^{N-1} a_{m} x^{m n}
$$

for some integer $n$ where $\operatorname{gcd}(n, N)=1$.

These are well-known operations and will preserve the properties of a sequence's periodic autocorrelation function. Using our program based on McLaughlin's algorithm described above, we arrived at our starting set of 20 sequences, from the original two, shown here:

$$
\begin{aligned}
& 0++-++-++++---+-+-+++----+--+-- \\
& 0+++-++---++++-+---++--++++-++ \\
& 0-+-+-++--+++----+-+++++-++-++ \\
& 0+++-++-+++--++++-++-----++-+-+ \\
& 0--++--+++-+++++-+--+++++--+-+- \\
& 0--+--+-++-+-++++++---+++-+++-+ \\
& 0-+-+--+++++--+-+++++-+++--++-- \\
& 0++-++-++++++-+----+++--++-+-+- \\
& 0+-+-++-----++-++++--+++-++-+++ \\
& 0+-+++-+++---++++++-+-++-+--+-- \\
& 0---+--+++----+-+++-+-++----+-- \\
& 0+-+-+--++---++++-+------+--+-- \\
& 0---+--+---++----+--+++++--+-+- \\
& 0++--++---+-----+-++-----++-+-+ \\
& 0--+----++-+-+++-+----+++--+--- \\
& 0++-++-+--+-+------+++---+---+- \\
& 0+-+-++-----++-+----+---++--++ \\
& 0--+--+------+-++++---++--+-+-+ \\
& 0-+-+--+++++--+----++---+--+--- \\
& 0-+---+---+++-----+-+--+-++-++
\end{aligned}
$$


Table 12 OHETSs starting versus ending set (period 31)

\begin{tabular}{llc}
\hline Measure & Starting set & Ending set \\
\hline Average GMF & 2.9640 & 5.3189 \\
Average SPAR & 2.7618 & 1.9463 \\
Average aperiodic cross-correlation & 3.0871 & 3.0452 \\
\hline
\end{tabular}

The result of our program for producing the ending set produces:

$$
\begin{aligned}
& ---++++-+---+-+--++++-++0+++-++ \\
& --+-++++++-++-++0-+-+-++--+++-- \\
& ++-+++--++++-+----++-++0+++- \\
& ++-+--+++++--+-+-0--++--++-+++ \\
& +-++-+-++++++---+++-+++-+0--+-- \\
& +++-+++--++--0-+-+--+++++--+-++ \\
& --+++--++-+-+-0++-++-++++++-+- \\
& -+++0+-+-++----++-++++--+++-+ \\
& --+--0+-+++-+++---+++++-+-++-+ \\
& +++----++++-+-++---+--0---+- \\
& ++-+-----+--+--0+-+-+--++--++ \\
& --+---++---+--+++++--+-+0---+ \\
& --+-++-----++-+-+0++--++---+-- \\
& --+---0--+---++-+-+++-+---+++ \\
& -+--+-+-----+++---+--+-0++-++ \\
& ---+---++-1+0+-+-++----++-+-- \\
& ++---++--+-+-10--+--+----+-+ \\
& +---0-+-+--+++++--+----++---+- \\
& ++-++0-+---+---+++-----+-+--+ \\
& ----+--+-0++-++-++++---++-++
\end{aligned}
$$

The relevant properties for these sets are as follows:

Thus, we are able to provide a set of 20 near-perfect (periodic side lobes are \pm 1 instead of 0) sequences with higher energy, lower SPAR, and comparable average GMF and cross-correlations using our OHETSs when compared to McLaughlin's example.

In the same patent, McLaughlin also gives an example of 12 perfect ternary sequences with the main lobe of size 16. His starting set is given by:

$$
\begin{aligned}
& --0-+00-++0000+-0+0+00+000+0++- \\
& --+-0++-000+0+0-+0+0000++00+00- \\
& 00-00-+0000-0++0-0+000+-+0++++-
\end{aligned}
$$


Table 13 McLaughlin starting versus ending set (perfect ternary sequences, period 31, main lobe 16)

\begin{tabular}{lll}
\hline Measure & Starting set & Ending set \\
\hline Average GMF & 2.5514 & 5.5013 \\
Average SPAR & 2.9595 & 2.1521 \\
Average aperiodic cross-correlation & 1.4409 & 1.3710 \\
\hline
\end{tabular}

$$
\begin{aligned}
& 00-0+-00+++-+000-+0+++0-0+0000- \\
& 0000-0+0--+0++000-+-++00++0+00- \\
& 0000+0-0+++0+-000+-+++00-+0-00- \\
& 00+00++0000+0+-0+0+000-++0-+--- \\
& 00+0++00++-+-000++0+--0+0-0000- \\
& ++0+-00+--0000++0-0-00+000+0++- \\
& ++0+000+00-0-0++0000--+00-+0++- \\
& ++0+000+00+0+0-+0000++-00+-0--- \\
& ++++0+-+000+0-0++0-0000+-00-00-
\end{aligned}
$$

and his ending set, after running through our program, gives:

$$
\begin{aligned}
& +0000+-0+0+00+000+0++---0-+00-+ \\
& ++00+00---+-0++-000+0+0-+0+0000 \\
& 00-0++0-0+000+-+0++++-00-00-+00 \\
& -0+-00+++-+000-+0+++0-0+0000-00 \\
& -+0++000-+-++00++0+00-0000-0+0- \\
& 00-0000+0-0+++0+-000+-+++00-+0- \\
& 0+0+-0+0+000-++0-+---00+00++000 \\
& -0+0-0000-00+0++00++-+-000++0+- \\
& +0++-++0+-00+--0000++0-0-00+000 \\
& +00-0-0++0000--+00-+0++-++0+000 \\
& +-00+-0---++0+000+00+0+0-+0000+ \\
& +-00-00-++++0+-+000+0-0++0-0000
\end{aligned}
$$

We attempt to better his set by taking two inequivalent perfect ternary sequences of period 31 with the main lobe 16 . These two sequences are given by

$$
[---0-+00-++0000+-0+0+00+000+0++]
$$

and

$$
[-00+00++0000+0+-0+0+000-++0-+--] .
$$


Using our method of finding all possible shifts, negations, and automorphisms, followed by removing any sequences which are cyclic shifts of another, we can create a starting set of 22 such perfect ternary sequences given by:

$$
\begin{aligned}
& 0++---0-+00-++0000+-0+0+00+000+ \\
& 0+-+0++++-00-00-+0000-0++0-0+00 \\
& 00-+-++00++0+00-0000-0+0--+0++0 \\
& 00+0-0++0-0000+-00-00-++++0+-+0 \\
& 00-00+0++00++-+-000++0+--0+0-00 \\
& 0--+++0+-00+--0000-+0-0-00-000- \\
& 0-+-0----+00+00+-0000+0--0+0-00 \\
& 00+-+--00--0-00+0000+0-0++-0--0 \\
& 00-0+0--0+0000-+00+00+----0-+-0 \\
& 00+00-0--00--+-+000--0-++0-0+00 \\
& 0000--+00-+0+++--0-000-00-0-0+- \\
& 0-+---00+00++0000+0+-0+0+000-++ \\
& 00+-+++00-+0-00-0000+0-0+++0+-0 \\
& 00+00-0-0++0000--+00-+0++-++0+0 \\
& 0000-00-0+-00+++-+000-+0+++0-0+ \\
& 0+--0000++0-0-00+000+0++-++0+-0 \\
& 0+-+++00-00--0000-00+0-0-000+-- \\
& 00-+---00+-0+00+0000-0+0---0-+0 \\
& 00-00+0+0--0000++-00+-0--+--0-0 \\
& 0000+00+0-+00---+-000+-0---0+0- \\
& 0-++0000--0+0+00-000-0--+--0-+0 \\
& 0-0000--00-00+++-+0--+000-0-0+-
\end{aligned}
$$

After this starting set is processed by our program, we get the following set of 22 perfect ternary sequences:

$$
\begin{aligned}
& +0000+-0+0+00+000+0++---0-+00-+ \\
& 00-0++0-0+000+-+0++++-00-00-+00 \\
& -+0++000-+-++00++0+00-0000-0+0- \\
& +-00-00-++++0+-+000+0-0++0-0000 \\
& -0+0-0000-00+0++00++-+-000++0+- \\
& -0000-+0-0-00-000-0--+++0+-00+- \\
& 00+0--0+0-000-+-0----+00+00+-00 \\
& +-0--000+-+--00--0-00+0000+0-0+ \\
& -+00+00+----0-+-000-0+0--0+0000 \\
& +0-0+0000+00-0--00--+-+000--0-+ \\
& -+00-+0+++-000-00-0-00+-0000-
\end{aligned}
$$


Table 14 Our starting versus ending set (perfect ternary sequences, period 31, main lobe 16)

\begin{tabular}{lll}
\hline Measure & Starting set & Ending set \\
\hline Average GMF & 3.6494 & 5.4460 \\
Average SPAR & 2.6113 & 2.1714 \\
Average aperiodic cross-correlation & 1.6100 & 1.3783 \\
\hline
\end{tabular}

$$
\begin{aligned}
& 0+0+-0+0+000-++0-+---00+00++000 \\
& 00-0000+0-0+++0+-000+-+++00-+0- \\
& 00+00-0-0++0000--+00-+0++-++0+0 \\
& -0+-00+++-+000-+0+++0-0+0000-000 \\
& +0++-++0+-00+--0000++0-0-00+000 \\
& 0-0-+0-0-000+--0+-+++00-00--000 \\
& 00+0000-0+0---0-+000-+---00+-0+ \\
& 00-00+0+0--0000++-00+-0--+--0-0 \\
& +0-+00---+-000+-0--0+0-0000+00 \\
& -0--+--0-+00-++0000--0+0+00-000 \\
& --00-00+++-+0--+000-0-0+-0-0000
\end{aligned}
$$

The relevant properties are given by Table 14 .

In this case, we achieve very similar values to what McLaughlin was able to get. However, we are able to introduce 10 more sequences into the set by looking for negations, shifts, and automorphisms of two inequivalent sequences, thus providing a larger set to work with.

\subsection{Modification to McLaughlin's algorithm}

Here, we describe our attempt to use a modified version of McLaughlin's algorithm above to generate "good" sets of high-energy sequences. We define a "good" set of sequences to have the properties:

1. Each sequence has a high GMF (a threshold GMF value must be defined).

2. The aperiodic autocorrelation side lobes of each sequence are small (see Eq. 17).

3. The sequences are pairwise orthogonal (See Proposition 4).

4. All sequences in the set are high in energy (we allow a single 0 entry).

To obtain such a set of sequences, we start with a sequence (of OHETS type) from the construction in Theorem 5. This sequence is turned into a set of $n$ sequences, where $n$ is the length of the sequence, by cyclic shifts. This will generate $n$ different sequences. From this set, we remove any sequences whose GMF is below a threshold value. Next, we further trim the list by choosing only sequences whose autocorrelations satisfy that their side lobes all lie at, or less than, $-13 \mathrm{~dB}$ below the main lobe. That is, we wish to have aperiodic autocorrelation side lobes satisfying, at worse: 


$$
10 \log \left(\frac{C_{\text {aper }}(i)}{C_{\text {aper }}(0)}\right) \leq-13
$$

where $i=1,2, \ldots, n-1$. The sequences which meet this condition are kept, the others are removed. The resulting set is our "good" set. We aim to generate sets where this $\mathrm{dB}$ threshold is $-15 \mathrm{~dB}$, but will accept a value as large as $-13 \mathrm{~dB}$.

Proposition 4 The nearly pairwise orthogonality of our "good" set is met when the set contains shifts of an OHETS sequence.

Proof Orthogonality is measured by the usual dot product of two sequences. Thus, we take two sequences in our set, say $S_{1}$ and $S_{2}$, and compute their dot product. This is equivalent to finding the main lobe of their cross-correlation. Since these two sequences are shifts of one another, then $S_{2}=S_{1} x^{t}$ for some integer $t$. Thus,

$$
S_{1} S_{2}=S_{1} S_{1} x^{t}
$$

It follows that the dot product will simply be a shift, by $t$ units, of any such sequence's autocorrelation. That is to say that the dot product of two such sequences will be a side lobe of their periodic ACF. By Theorem 5, all periodic side lobes, of a given OHETS, are -1 . Hence, the dot product of two such sequences is -1 .

Remark 1. Strict orthogonality is when the dot product is 0 , but for our sequences this dot product is guaranteed to always be -1 , regardless of length. Thus, as the sequence length increases, the set of sequences become closer to the ideal definition of pairwise orthogonality in an asymptotic sense.

2. The condition of Eq. (17) can be restated in terms of a fraction of the sequence length as it implies that $C_{\text {aper }}(i)=10^{-1.3} C(0)=\frac{n-1}{10^{1.3}} \approx \frac{n-1}{20}$. Thus, we are looking for side lobes which are no larger than $\frac{1}{20}$ of the main lobe's value.

\subsubsection{Tables of some good high-energy sets}

The following tables will provide examples of our so-called good sets from the previous section. We specifically have run exhaustive searches for where the GMF is no lower than 5 and the autocorrelation ratios are no higher than $-13 \mathrm{~dB}$. For lengths where multiple good sets are found, we show the one with the smallest autocorrelation ratios. Due to the length of the sequences, we compact them by writing them in hexadecimal format. To achieve this, we follow these steps:

1. Take the first sequence in the set and find the single 0 value.

2. Replace all $-1 \mathrm{~s}$ in this sequence by 0 .

3. Convert this binary $\{0,1\}$ sequence to a hexadecimal form by using MATLAB's binaryVectorToHex() function.

4. Find the zero locations of the remaining sequences in the set to determine how many positions they are right-cyclically shifted from the first.

Using this process allows us to present only the first sequence in the set. By reversing the process, one can generate the entire set (convert the starting sequence back to binary 
Table 15 Starting sequences for good high-energy sets

\begin{tabular}{ll}
\hline Length & Starting sequence (hex) \\
\hline 181 & 1B1508FD1D5C5F88546F3EC99AD251E1021E292D664DF3 \\
251 & 5916C4D79D95D22B230A6E4BB27D6038E2121F2950883EEF56B07B7B8E3F941 \\
509 & 1BD68BFEDE8FC0B750F08E3C19ECD29CA72966F3078E21E15DA07E2F6FFA2D7 \\
& B9232657580A76EAB0C8088E5E693A2CA5345C967A7110130D576E501AEA64C49 \\
521 & 07BAF6477E451B05A34EBEB531A6752F36D9E95CCB195AFAE58B41B144FDC4DEB \\
& BC8479221C58112985FD17030D3FC4D475AE2B23FCB0C0E8BFA194881A38449E21 \\
0B97207700E564168D4BC2A3035D79DF2E3176CA45D27B475716F25D129B7463 & A7DCF5D6062A1E958B4135380770274EBB5BC99EB41DA775ED873FA2923394AB \\
& EA0246BF9E577C4C80C6659168F0F459C67EE020DA1743240930BA16C101DF98 \\
& E68BC3C5A26998C04C8FBA9E7F589015F54A7312517F386DEBB96E0B5E64F6B7 \\
& 02A9C54A5CF2D11F04D981AE7392C04D8F85F349F3D5C299F51CB49D94F4D5CD \\
& D5D22A262A686B236963A8335E2A1836982F0726FE5B18C53F326F83BA5862D6 \\
& AE355FF84A28304578D30AD7A0E8CA110F0B3D2013FE471328D2ED2B74CC2620 \\
& 7A0998910403FDF76E66FA1FB9BCCD12B48B4EB371D8037FB432F0F77ACE8FA1 \\
& 4AF34E15DF3EBADE0 \\
& 04A8FCE0F8A3909A12E4AE7ED7BE713E3D689E520FD4A8B83BB3B62C73DE7D1 \\
& D9AC4E3B9F7596D8AE37B49AF5CBCBBF01161628536909C5724B288311C6E53 \\
& 23A0C218E5C91911F1756A07DAC374A1C1B8C10A40C56C5BD37B1D707C60756 \\
& FB027E732C9D006FB2CCE00B7A71D953A4528E80F04705CD6445F53778E5338 \\
& AA64B7FDB06E72B406BF52C102A232CFBCB444C7EAD2F1B48F483A3051128BB \\
& DB59E1B0F811558B7BDD2A57F6C2764F9971660D91BC9015AB44212E5577E0F \\
& 278652422EB775F3A3ED0ED270B4A81CDDD2C20CB3BABF7CB5029FD2B189F24 \\
& 012D9AAE3358E113505DD94C5F1DF0FE8EB5DA356471A12FF8CCB209FF46CB3 \\
& 181BF2 \\
\hline &
\end{tabular}

form, replace all 0 s with -1 , puncture the sequence in its "zero location," and then generate the other by cyclically shifting to the right. ). Two tables are presented. The first gives the starting sequence for each length, and the second gives the remaining information (Tables 15 and 16).

All shifts for length 1021:

0123456789101112131415161718192021222324252627282930 313233343536373839404142434445464748495051525354555657 585960616263646566676869707172737475767778798081828384 85868788919295969899117118119120121122123124125513514515 516517518519520521539540542543546547550551552553554555556 557558559560561562563564565566567568569570571572573574575 576577578579580581582583584585586587588589590591592593594 595596597598599600601602603604605606607608609610611612613 614615616617618619620621622623624625626627628629630631632 633634635636637638 
Table 16 Information for good high-energy sets

\begin{tabular}{llllll}
\hline Length & Set size & Min GMF & Max corr ratio $(\mathrm{dB})$ & Zero position & Shifts \\
\hline 181 & 4 & 5.2976 & -13.0103 & 38 & $0,10,96,106$ \\
251 & 4 & 5.0233 & -13.1876 & 52 & $0,26,122,148$ \\
509 & 8 & 6.0032 & -14.5059 & 127 & $0,1,2,4,252,254,255,256$ \\
521 & 2 & 6.0068 & -15.1188 & 133 & 0,256 \\
1021 & 208 & 5.0204 & -15.0345 & 192 & See Below \\
1091 & 8 & 6.0017 & -16.0606 & 268 & $0,1,2,5,551,554,555,556$ \\
2039 & 504 & 5.0004 & -16.1023 & 380 & See Below \\
\hline
\end{tabular}

All shifts for length 2039:

0123456789101112131415161718192021222324252627282933 414243444546474849505152535455565758596061626364656667 686970717273747576777879808182838485868788899091929394 9596979899100101102103104105106107108109110111112113114115 116117118119120121122123124125126127128129130131132133134 135136137138139140141142143144145146147148149150151152153 154155156157158159160161162163164165166167168169170171172 173174175176177178179180181182183184185186187188189190191 192193194195196197198199200201202203204205206207208209210 211212213214215216217218219220221222223224225226227228229 230231232233234235236237238239240241242243244245246247248 24925025125225325425525625725825926026110191020102110221023 102410251026102710281029103010311032103310341035103610371038 103910401041104210431044104510461047104810491050105110521053 105410551056105710581059106010611062106310641065106610671068 106910701071107210731074107510761077107810791080108110821083 108410851086108710881089109010911092109310941095109610971098 109911001101110211031104110511061107110811091110111111121113 111411151116111711181119112011211122112311241125112611271128 112911301131113211331134113511361137113811391140114111421143 114411451146114711481149115011511152115311541155115611571158 115911601161116211631164116511661167116811691170117111721173 117411751176117711781179118011811182118311841185118611871188 118911901191119211931194119511961197119811991200120112021203 120412051206120712081209121012111212121312141215121612171218 121912201221122212231224122512261227122812291230123112321233 123412351236123712381239124712511252125312541255125612571258 125912601261126212631264126512661267126812691270127112721273 1274127512761277127812791280

An exhaustive computer search was used to produce all possible sets of OHETS sequences up to length 2039. Such sets have been created computationally for all prime lengths up to 2048. The above sets were chosen to display because: 
- Length 181 is the first set with a maximum correlation ratio of $-13 \mathrm{~dB}$.

- Lengths 251, 509, 1021, and 2039 are near powers of 2.

- Length 521 is the first set with a maximum correlation ratio of $-15 \mathrm{~dB}$.

- Length 1091 is the first set with maximum correlation ratio of $-16 \mathrm{~dB}$.

Remark The above sets would be computationally challenging to get without the theoretical results from Theorem 5. In fact, the theorems presented in this paper were generated after doing exhaustive computational searches for preferable sequences and observing patterns found within them. These initial searches only included lengths up through 32 as the computational complexity grows rapidly. Such searches require the generation and examination of all $2^{n-1}$ possible sequences of each length $n$. Once generated, each sequence's autocorrelation function must be calculated, further slowing the process. The theorems above allow for these sets to be generated nearly instantaneously (relatively speaking).

\section{Application of OHETSs to frame synchronization}

Synchronization technology is an indispensable tool in wireless communication. Good metrics to evaluate the performance of synchronization include: the probability of missing synchronization and false synchronization, and frame synchronization time. In this section, we utilize our new sequences and compare their synchronization performance with known sequences including the well-known Barker codes. The simulation results show that the new sequences do out-perform their previously known compeers.

Frame synchronization is a method used in digital communication systems to find valid data in a transmission that consists of data frames. To realize frame synchronization, the transmitter inserts a fixed data pattern at the start of each data frame to mark the start of valid data. The receiver searches for the fixed pattern in each data frame and achieves frame synchronization when the correlation between the input data and the fixed pattern is ideal. The probability of missing synchronization and false synchronization, and frame synchronization time are the main factors to influence the performance of the synchronization. See [17] and [8] for more information. For some recent results on comparative analysis of performance of different codes/sequences for frame synchronization based on probability of missing synchronization, see [2].

A MATLAB program was used to simulate the chance of missed synchronization from 0 to $12 \mathrm{~dB}$ in steps of $0.5 \mathrm{~dB}$. For each step, a simulation of size 10,000 was used in a Monte Carlo approach. The sequences being compared are in the following table followed by the results.

As Figs. 2 and 3 show, the OHETSs offer performance advantages over both the Barker sequence and the perfect pair sequences of equal lengths (Table 17). This advantage is seen throughout the $\mathrm{dB}$ range where the OHETS consistently give a lower probability of missed synchronization. This suggests promising applications of these new OHETS in wireless communication systems. 


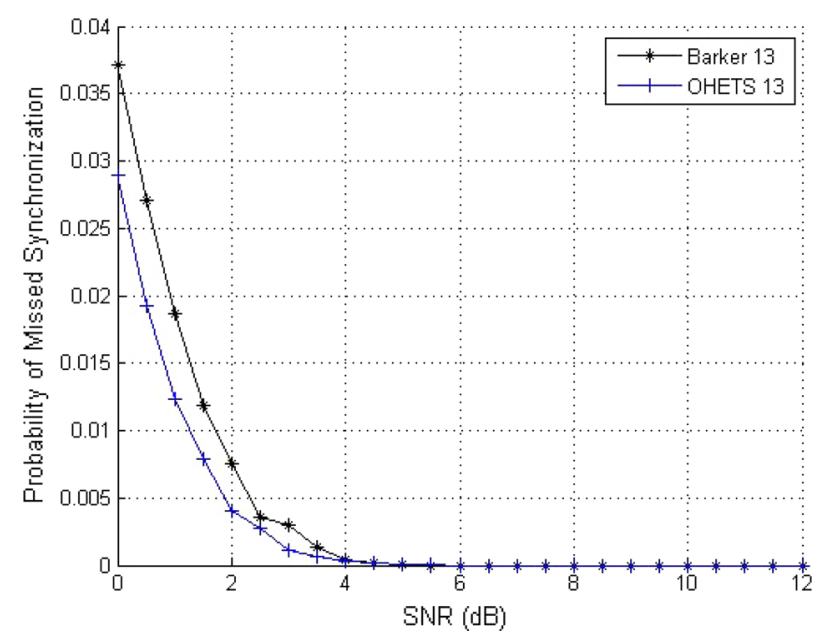

Fig. 2 Frame synchronization of length 13 OHETS and Barker codes

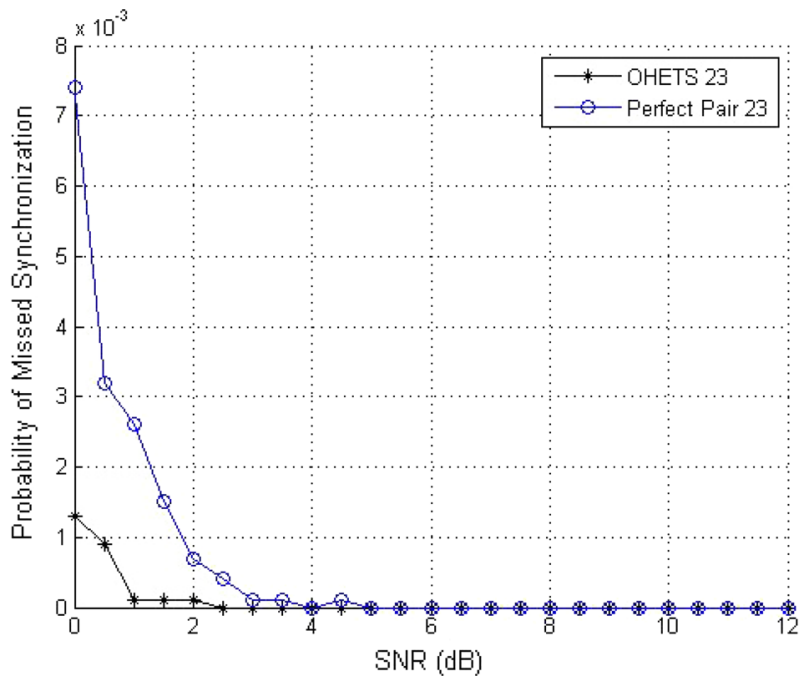

Fig. 3 Frame synchronization of length 23 OHETS and perfect pair

Table 17 Sequences compared for frame synchronization

\begin{tabular}{ll}
\hline Name & Sequence \\
\hline Barker length 13 & {$[+++++--++-+-+]$} \\
OHETS length 13 & {$[0+-++----++-+]$} \\
OHETS length 23 & {$[0++++-+-++--++--+-+----]$} \\
Perfect pair length 23 & $A=[+----+-+--++--++-+-++++]$ \\
& $B=[+0000+0+00++00++0+0++++]$
\end{tabular}


Table 18 Optimal sequences of prime lengths with two zeros

\begin{tabular}{ll}
\hline Length & Sequence \\
\hline 4 & {$[+-00]$} \\
5 & {$[+--00]$} \\
6 & {$[+-0--0]$} \\
7 & {$[+---0-0]$} \\
8 & {$[+--0+0-]$} \\
9 & {$[+----0+0-]$} \\
11 & {$[+----+0-+0]$} \\
13 & {$[+-----+-+0-0+]$} \\
15 & {$[+----00-+-+--+]$} \\
16 & {$[+----0+--0+-+-+]$} \\
25 & {$[+0++0----++-+++-+++-+-++-]$} \\
27 & {$[+0++0---+-+--+++-+++++-+--+]$}
\end{tabular}

\section{Remarks and problems}

Remark 1. We also have found by computer search some sporadic examples of lengths 9 and 15 all of whose side lobes are 1 or -1 , making these optimal. The sequences are given by $[+----+-0+]$ and $[+------+-++--+0]$, respectively.

2. By allowing exactly two zeros, we obtain further examples of such ternary sequences of lengths $4,5,6,7,8,9,11,13,15,16,25,27$ by computer search. The following table contains such examples by length (Table 18):

3. We believe allowing for more zeros would give rise to more such sequences.

4. Circulant weighing matrices (also known as Ipotov's ternary sequences) are infinite families of such sequences whose lengths are $\frac{q^{n}-1}{q-1}$ where $q$ is any prime power, $n>1$ is any odd integer, and the number of zeros in the sequence is $\frac{q^{n-1}-1}{q-1}$.

5. Ipotov's sequences are "perfect," in the sense that all side lobes are zero.

\section{Problems}

1. Generalize the sporadic examples found of lengths 9 and 15 (with a single zero) to infinite families.

2. Obtain at least one infinite family of such sequences with exactly two zeros.

\section{References}

1. Arasu, K.T.: Sequences and arrays with desirable correlation properties. In: Crnković, D., Tonchev, V. (eds.) Information Security, Coding Theory and Related Combinatorics, NATO Science for Peace and Security Series D: Information and Communication Security, vol. 29, pp. 136-171. IOS Press, Amsterdam (2011) 
2. Arasu, K. T., Arya, D., Kedia, D.: Comparative analysis of punctured sequence pairs for frame synchronization applications. In: IEEE International Conference on Computational Techniques in Information and Communication Technologies (ICCTICT), pp. 470-475. (2016)

3. Arasu, K.T., Jungnickel, D., Ma, S.L., Pott, A.: Strongly regular cayley graphs with $\lambda-\mu=-1$. J. Combin. Theory Ser. A 67(1), 116-125 (1994)

4. Camion, P., Mann, H.B.: Antisymmetric difference sets. J. Number Theory 4, 266-268 (1972)

5. Farnett, E.C.: Pulse Compression Radar, 2nd edn. McGraw-Hill, New York (1990)

6. Ipatov, V.P.: Ternary sequences with ideal autocorrelation properties. Radio Eng. Electron Phys. 24, 94-97 (1979)

7. Kolman, J.: Aperture weighting for maximum contrast of SAR imagery. In: 2008 IEEE Radar Conference, pp. 1-6. (2008)

8. Luke, H.D.: Mismatched filtering of periodic quadriphase and 8-phase sequences. IEEE Trans. Commun. 51(7), 1061-1063 (2003)

9. McLaughlin, M.: Method and apparatus for generating codewords. US Patent US 7,787,544 B2, Delaware Limited, Dublin IE, Aug 2010

10. Mileski, A.: Periodic sequences with optimal properties for channel estimation and fast start-up equalization. IBM J. Res. Dev. 27, 426-431 (1983)

11. Paley, R.E.A.C.: On orthogonal matrices. J. Math. Phys. 12, 311-320 (1933)

12. Setlur, P., Hollon, J., Arasu, K. T., Rangaswamy, M.: On a formal measure of doppler tolerance. In: 2017 IEEE Radar Conference (RadarConf), pp. 1751-1756. Seattle, WA (2017)

13. Simon, M.K.: Spread spectrum communications. Comput. Sci. Press 1, 666-673 (1985)

14. Tirkel, A.Z., Rankin, G.A., Van Schyndel, R.M.: Electronic water mark. DICTA 93, 666-673 (1993)

15. Van Schyndel, R.G.: Using phase-modulated probe signals to recover delays from higher order nonlinear systems. In: 2001 IEEE Engineering in Medicine and Biology: Biomedical Research, pp. 94-97 (2001)

16. Xu, L.: Phase coded waveform design for sonar sensor network. In: Conference on Communications and Networking in China, pp. 251-256. (2011)

17. Zhao, Z., Zhang, J.: Research of frame synchronization technology based on perfect punctured binary sequence pairs. Rev. Adv. Mater. Sci. 33, 261-265 (2013) 